\title{
The dynamic relaxation method using new formulation for fictitious mass and damping
}

\author{
M. Rezaiee-pajand" and J. Alamatian \\ Department of Civil Engineering, Ferdowsi University of Mashhad, Mashhad, P.O. Box. 91775-1111, Iran
}

(Received July 5, 2008, Accepted October 6, 2009)

\begin{abstract}
This paper addresses the modified Dynamic Relaxation algorithm, called mdDR by minimizing displacement error between two successive iterations. In the mdDR method, new relationships for fictitious mass and damping are presented. The results obtained from linear and nonlinear structural analysis, either by finite element or finite difference techniques; demonstrate the potential ability of the proposed scheme compared to the conventional DR algorithm. It is shown that the mdDR improves the convergence rate of Dynamic Relaxation method without any additional calculations, so that, the cost and computational time are decreased. Simplicity, high efficiency and automatic operations are the main merits of the proposed technique.
\end{abstract}

Keywords: dynamic relaxation; error minimizing; fictitious mass and damping; nonlinear analysis.

\section{Introduction}

Final stage of any structural analysis is performed by solving a simultaneous system of equations resulting from finite element or finite difference scheme as

$$
[S]\{D\}=\{f\}=\{P\}
$$

where $[S]$ is structural stiffness matrix, $\{D\},\{f\}$ and $\{P\}$ are displacement, internal force and external load vectors, respectively. In nonlinear behaviors, such as geometrical, material or contact nonlinearity, internal force vector is nonlinear function of displacement. The most common methods for solving Eq. (1) in nonlinear analysis are iterative procedures. These techniques can be classified as implicit or explicit schemes (Felippa 1999). Explicit methods use residual force to get answer, so that all calculations are performed by vector operations. Simplicity and high efficiency in nonlinear analysis are the main specifications of the explicit techniques. On the other hand, implicit procedures are formulated based on residual force derivatives (stiffness matrix). Because of using matrix operations, calculations of implicit methods are complex and time consuming. For example, snap through or snap back points, in which stiffness matrix is zero or undefined, cause some difficulties. However, convergence rate of the implicit methods is greater than the explicit schemes.

Here, Dynamic Relaxation (DR) method is utilized for solving Eq. (1). This is an explicit iterative

$\dagger$ Professor, Corresponding author, E-mail: mrpajand@yahoo.com

†Ph.D. Student, E-mail: alamatian@yahoo.com 
procedure, in which static system is transferred to an artificial dynamic space by adding artificial inertia and damping forces

$$
[M]^{n}\{\ddot{D}\}^{n}+[C]^{n}\{\dot{D}\}^{n}+[S]^{n}\{D\}^{n}=\{f\}^{n}=\{P\}^{n}
$$

where, $[M]^{n}$ and $[C]^{n}$ are fictitious mass and damping matrices in the $\mathrm{n}^{\text {th }}$ iteration of DR algorithm, respectively, and assumed to be diagonal. Also, super dots denote derivatives related to the fictitious time. In deed, Dynamic Relaxation method calculates steady state response of dynamic system (2) as answer of Eq. (1), using numerical techniques. This method which has been introduced by Otter (1966) or Day (1965) can be explained by either mathematical or physical theories. Mathematically, DR formulation is based on the second order Richardson rule, developed by Frankel (1950). Heat transfer problem in rectangular region is an example of this point of view. Physically, DR scheme can be illustrated by steady state response of an artificial dynamic system. Due to this theory, fictitious density was introduced by Welsh (1967) and Cassell (1968).

First DR application in nonlinear analysis was performed by Rushton (1968). Brew and Brotton formulated Dynamic Relaxation method using first order dynamic equilibrium relationship, in which stability conditions of frame structures were studied (Brew and Brotton 1971). Simultaneously, Wood defined fictitious mass by using upper bound of spectral radius of the coefficients matrix (Wood 1971). He showed that the convergence rate of DR is higher than the semi iterative procedures in linear analysis. Also, estimation of critical damping was obtained by Bunce (1972). Alwar and his coworkers determined steady state response from an exponential function (Alwar et al. 1975). Furthermore, Cassell and Hobbs utilized Gerschgörin circle theory for fictitious mass values and applied this method to nonlinear problems (Cassell and Hobbs 1976).

Frieze and his coworkers used DR algorithm for nonlinear analysis of plates (Frieze et al. 1978). The first related error analysis was done by Papadrakakis (1981). He suggested an automatic procedure for selection of DR parameters. Another explicit formulation was performed by Underwood (1983). Also, implicit DR relationships were introduced by Felippa (1982). Zienkiewicz et al. suggested an accelerated procedure for improvement of the convergence rate (Zienkiewicz and Lohner 1985). By using weighting factors for mass and damping, Al-Shawi and Mardirosian utilized Dynamic Relaxation for finite element analysis of bending plates (Shawi and Mardirosion 1987). Qiang determined fictitious time and damping by Rayleigh's principle (Qiang 1988). The maDR algorithm was proposed by Zhang in which estimation of steady state response was modified (Zhang and Yu 1989).

Other researchers such as, Turvey et al. (1990) and Bardet et al. (1991) studied some applications of DR method. The first use of DR scheme in problems with snap through or snap back points was performed by Ramesh and Krishnamoorthy $(1993,1994)$ They combined DR algorithm by arc length procedure. On the other hand, Kadkhodayan and coworkers introduced new model for fictitious damping (Zhang et al. 1994). Also, they utilized Dynamic Relaxation method in elasticplastic and plate buckling problems (Kadkhodayan and Zhang 1995, Kadkhodayan et al. 1997). Nonlinear analysis of buckling propagation in pipe lines was performed by Pasqualinoe and Estefen, Simultaneously (Pasqualino and Estefan 2001). Wood (2002) and Han (2003) used DR method for shape finding analysis. Recently, Dynamic Relaxation was successfully applied to linear and nonlinear analysis of composite structures (Turvey and Salehi 2005). Moreover, Dynamic Relaxation method has an ability to use in nonlinear dynamic analysis of structures so that the errors are reduced (Rezaiee-Pajand and Alamatian 2008).

In the explicit DR method, fundamental relationships are formulated by using central finite 
difference as below (Zhang and Yu 1989)

$$
\begin{gathered}
\dot{D}_{i}^{n+\frac{1}{2}}=\frac{2 m_{i i}-\tau^{n} c_{i i}}{2 m_{i i}+\tau^{n} c_{i i}} \dot{D}_{i}^{n-\frac{1}{2}}+\frac{2 \tau^{n}}{2 m_{i i}+\tau^{n} c_{i i}} r_{i}^{n} \quad i=1,2, \ldots, q \\
D_{i}^{n+1}=D_{i}^{n}+\tau^{n+1} \dot{D}_{i}^{n+\frac{1}{2}} \quad i=1,2, \ldots, q
\end{gathered}
$$

where, $\tau^{n}, m_{i i}$ and $c_{i i}$ are fictitious time step, $\mathrm{i}^{\text {th }}$ diagonal element of fictitious mass and damping matrices in the $\mathrm{n}^{\text {th }}$ iteration of DR, respectively. Also, $q$ denotes the number of degrees of freedom and $r_{i}^{n}$ is the residual force of the $\mathrm{i}^{\text {th }}$ degree of freedom in the $\mathrm{n}^{\text {th }}$ iteration

$$
\{R\}^{n}=[M]^{n}\{\ddot{D}\}^{n}+[C]^{n}\{\dot{D}\}^{n}=\{P\}^{n}-\{f\}^{n}
$$

DR iterations are unstable because numerical time integration is used to integrate the differential equations of motion. Hence, fictitious parameters such as time step, diagonal mass and damping matrices are determined so that the stability conditions are satisfied and the convergence rate is increased.

In this paper, new fictitious mass and damping matrices are formulated by minimizing errors in DR iterations. Then, the mdDR algorithm is presented based on the suggested formulation. Finally, some linear and nonlinear problems are analyzed by either finite element or finite difference procedures. These solutions are utilized for numerical verification of the proposed method.

\section{The error analysis of dynamic relaxation method}

To study stability of DR algorithm, error analysis between two successive iterations should be performed. Substituting Eq. (3) into (4) and using the previous velocity $\left(\dot{D}_{i}^{n-1 / 2}\right)$ as a function of displacement, leads to the following relationship

$$
D_{i}^{n+1}=D_{i}^{n}+\frac{\tau^{n+1}}{\tau^{n}} \frac{2 m_{i i}-\tau^{n} c_{i i}}{2 m_{i i}+\tau^{n} c_{i i}}\left(D_{i}^{n}-D_{i}^{n-1}\right)+\frac{2 \tau^{n+1} \tau^{n}}{2 m_{i i}+\tau^{n} c_{i i}}\left(p_{i}-\sum_{j=1}^{q} S_{i j}^{n} D_{j}^{n}\right) \quad i=1,2, \ldots, q
$$

Displacement error in the $\mathrm{n}^{\text {th }}$ iteration is defined as follows (Undewood 1983)

$$
\{e\}^{n}=\{D\}^{n}-\{D\}^{*}
$$

where, $\{D\}^{*}$ and $\{e\}^{n}$ are answer of Eq. (1) and displacement error in the $\mathrm{n}^{\text {th }}$ iteration, respectively. By combining Eqs. (6) and (7), fundamental relationship of DR error can be simplified as below

$$
\{e\}^{n+1}=\{e\}^{n}+[A]^{n}\left(\{e\}^{n}-\{e\}^{n-1}\right)-[B]^{n}[S]^{n}\{e\}^{n}
$$

Here, $[A]^{n}$ and $[B]^{n}$ are diagonal matrices with the following entries

$$
\begin{gathered}
a_{i i}=\frac{\tau^{n+1}}{\tau^{n}} \frac{2 m_{i i}-\tau^{n} c_{i i}}{2 m_{i i}+\tau^{n} c_{i i}} \quad i=1,2, \ldots, q \\
b_{i i}=\frac{2 m_{i i}+\tau^{n} c_{i i}}{2 \tau^{n+1} \tau^{n}} \quad i=1,2, \ldots, q
\end{gathered}
$$


The DR iterations are stable if errors become near zero. For simplicity, the variation of the errors between two successive iterations is assumed to be linear (Undewood 1983)

$$
e_{i}^{n+1}=k_{i} e_{i}^{n}, \quad e_{i}^{n-1}=\frac{e_{i}^{n}}{k_{i}} \quad i=1,2, \ldots, q
$$

In which, $k_{i},(i=1,2, \ldots, q)$ is the error factor that controls stability and convergence rate. Substituting Eq. (11) into (8), gives the following equation

$$
k_{i}^{2}-\left(1+a_{i i}-\lambda_{i}^{B S}\right) k_{i}+a_{i i}=0 \quad i=1,2, \ldots, q
$$

Here, $\lambda_{i}^{B S}$ is the $i^{\text {th }}$ eigenvalue of $[B]^{-1}[S]$. For optimum convergence (minimum value of $k_{i}$, $i=1,2, \ldots, q)$, discriminant of Eq. (12) should be zero

$$
\Delta_{i}=0 \rightarrow\left(1+a_{i i}-\lambda_{i}^{B S}\right)^{2}=4 a_{i i} \quad i=1,2, \ldots, q
$$

Eq. (13) is the most important condition for stability and optimum convergence rate of the explicit DR method. This equation has real answers if expression in the right hand side is greater than or equal to zero

$$
a_{i i} \geq 0 \rightarrow-\frac{2}{\tau^{n}} m_{i i} \leq c_{i i} \leq \frac{2}{\tau^{n}} m_{i i} \quad i=1,2, \ldots, q
$$

Hence, two values for each eigenvalue of $[B]^{-1}[S]$ are calculated

$$
\begin{array}{ll}
\lambda_{i L}^{B S}=1+a_{i i}-2 \sqrt{a_{i i}} & i=1,2, \ldots, q \\
\lambda_{i U}^{B S}=1+a_{i i}+2 \sqrt{a_{i i}} & i=1,2, \ldots, q
\end{array}
$$

Here, $\lambda_{i L}^{B S}$ and $\lambda_{i U}^{B S}$ are lower and upper bounds of the $\mathrm{i}^{\text {th }}$ eigenvalues of $[B]^{-1}[S]$, respectively, which satisfy stability condition. Because of unknown parameters (fictitious mass, damping and time step) eigenvalues of $[B]^{-1}[S]$ can not be determined analytically. Hence, Gerschgörin's circles theory which is an approximate method is used to estimate the $\mathrm{i}^{\text {th }}$ eigenvalue of $[B]^{-1}[S]$ as follows (Undewood 1983)

$$
\left|\lambda_{i}^{B S}-\frac{2 \tau^{n+1} \tau^{n}}{2 m_{i i}+\tau^{n} c_{i i}} S_{i i}\right| \leq \frac{2 \tau^{n+1} \tau^{n}}{2 m_{i i}+\tau^{n} c_{i i}} \sum_{j=1}^{q}\left|S_{i j}\right| \quad i=1,2, \ldots, q
$$

Inequality (17) displays that each eigenvalue of $[B]^{-1}[S]$ can be lied in two discrete zones (I and II)

$$
\begin{gathered}
\lambda_{i}^{B S} \in I \Rightarrow \frac{2 \tau^{n+1} \tau^{n}}{2 m_{i i}+\tau^{n} c_{i i}} S_{i i} \leq \lambda_{i}^{B S} \leq \frac{2 \tau^{n+1} \tau^{n}}{2 m_{i i}+\tau^{n} c_{i i}} \sum_{j=1}^{q}\left|S_{i j}\right| \quad i=1,2, \ldots, q \\
\lambda_{i}^{B S} \in I I \Rightarrow \frac{2 \tau^{n+1} \tau^{n}}{2 m_{i i}+\tau^{n} c_{i i}}\left[S_{i i}-\sum_{\substack{j=1 \\
j \neq i}}^{q} \mid S_{i j}\right] \leq \lambda_{i}^{B S} \leq \frac{2 \tau^{n+1} \tau^{n}}{2 m_{i i}+\tau^{n} c_{i i}} S_{i i} \quad i=1,2, \ldots, q
\end{gathered}
$$

At this stage, it must be clear that which of these zones is more compatible with the structural specifications. For this purpose, the error factor $\left(k_{i}\right)$ is calculated from Eq. (12) 


$$
k_{i}=\frac{1+a_{i i}-\lambda_{i}^{B S}}{2} \quad i=1,2, \ldots, q
$$

The optimum convergence rate is obtained when $k_{i}=0, i=1,2, \ldots, q$. Using this condition and substituting $a_{i i}$ from Eq. (9) leads to the following result

$$
k_{i}=0 \rightarrow m_{i i}=\left(\frac{\tau^{n} \lambda_{i}^{B S}+\tau^{n+1}-\tau^{n}}{\tau^{n}+\tau^{n+1}-\tau^{n} \lambda_{i}^{B S}}\right) \frac{\tau^{n} c_{i i}}{2} \quad i=1,2, \ldots, q
$$

Eq. (21) must satisfy condition (14). Therefore the following condition is required

$$
\frac{\tau^{n} \lambda_{i}^{B S}+\tau^{n+1}-\tau^{n}}{\tau^{n}+\tau^{n+1}-\tau^{n} \lambda_{i}^{B S}} \geq 1 \rightarrow \lambda_{i}^{B S} \geq 1 \quad i=1,2, \ldots, q
$$

Moreover, expression in the left side of Eq. (22) should be non-negative. By sign determination of this relationship it is concluded

$$
\frac{\tau^{n+1}-\tau^{n}}{\tau^{n}} \leq \lambda_{i}^{B S} \leq \frac{\tau^{n+1}+\tau^{n}}{\tau^{n}} \stackrel{\tau^{n+1} \approx \tau^{n}}{\longrightarrow} \quad 0 \leq \lambda_{i}^{B S} \leq 2 \quad i=1,2, \ldots, q
$$

If inequality (23) is combined with (22), the following result is obtained

$$
1 \leq \lambda_{i}^{B S} \leq 2 \quad i=1,2, \ldots, q
$$

Inequality (24) determines upper and lower bounds of each eigenvalue of $[B]^{-1}[S]$ in which stability is guaranteed and convergence rate is also maximized. Applying these limits into the Eqs. (18) and (19) leads to the following results

$$
\begin{gathered}
\frac{2 \tau^{n+1} \tau^{n}}{2 m_{i i}+\tau^{n} c_{i i}} S_{i i} \geq 1, \quad \frac{2 \tau^{n+1} \tau^{n}}{2 m_{i i}+\tau^{n} c_{i i}} \sum_{j=1}^{q}\left|S_{i j}\right| \leq 2 \quad i=1,2, \ldots, q \quad \lambda_{i}^{B S} \in I \\
\frac{2 \tau^{n+1} \tau^{n}}{2 m_{i i}+\tau^{n} c_{i i}}\left[S_{i i}-\sum_{\substack{j=1 \\
j \neq i}}^{q}\left|S_{i j}\right|\right] \geq 1, \quad \frac{2 \tau^{n+1} \tau^{n}}{2 m_{i i}+\tau^{n} c_{i i}} S_{i i} \leq 2 \quad i=1,2, \ldots, q \quad \lambda_{i}^{B S} \in I I
\end{gathered}
$$

The above relationships can be simplified as follows

$$
\begin{gathered}
\sum_{j=1}^{q}\left|S_{i j}\right| \leq \frac{2 m_{i i}+\tau^{n} c_{i i} 2 \tau^{n+1} \tau^{n}}{2 \tau^{n+1} \tau^{n}} \leq 2 S_{i i} \quad i=1,2, \ldots, q \quad \lambda_{i}^{B S} \in I \\
S_{i i} \leq \frac{2 m_{i i}+\tau^{n} c_{i i} 2 \tau^{n+1} \tau^{n}}{2 \tau^{n+1} \tau^{n}} \leq 2\left[S_{i i}-\sum_{\substack{j=1 \\
j \neq i}}^{q}\left|S_{i j}\right|\right] \quad i=1,2, \ldots, q \quad \lambda_{i}^{B S} \in I I
\end{gathered}
$$

As a result, there is acceptable domain for fictitious parameters of DR if the stiffness elements of structure satisfy the following conditions

$$
S_{i i} \geq \sum_{\substack{j=1 \\ j \neq i}}^{q}\left|S_{i j}\right| \quad i=1,2, \ldots, q \quad \lambda_{i}^{B S} \in I
$$




$$
S_{i i} \geq 2 \sum_{\substack{j=1 \\ j \neq i}}^{q}\left|S_{i j}\right| \quad i=1,2, \ldots, q \quad \lambda_{i}^{B S} \in I I
$$

Mathematically, acceptable domain of Eq. (29) is greater than Eq. (30). Therefore, probability of the numerical instabilities decreases when Eq. (18) is used for eigenvalues of $[B]^{-1}[S]$. In the other words, boundaries of Eq. (19) may be ineffective and unsuitable. Moreover, in each row of the linear structural stiffness matrix, the diagonal entry is greater than or equal to the absolute sum along the rest of the row. Eq. (29) displays this specification. Therefore, Eq. (30) is not valid in linear structural analysis. Nevertheless, similar discussion may not be possible for nonlinear analysis because of variety of such behaviors. In this case, both Eqs. (29) and (30) have the same probability of validity. But, wide range of numerical studies indicates that DR iterations are unstable when eigenvalues are assumed in zone II (Eq. (19) or condition (30)). The presented discussions show that each eigenvalue of $[B]^{-1}[S]$ must be assumed in domain I, distinguished by Eq. (18). The paper uses this domain in DR formulation. By substituting $\lambda_{i L}^{B S}$ and $\lambda_{i U}^{B S}$ from Eqs. (15) and (16) into the inequality (18), the following results are achieved

$$
\begin{aligned}
& 1+a_{i i}-2 \sqrt{a_{i i}} \geq \frac{2 \tau^{n+1} \tau^{n}}{2 m_{i i}+\tau^{n} c_{i i}} S_{i i} \quad i=1,2, \ldots, q \\
& 1+a_{i i}+2 \sqrt{a_{i i}} \leq \frac{2 \tau^{n+1} \tau^{n}}{2 m_{i i}+\tau^{n} c_{i i}} \sum_{j=1}^{q}\left|S_{i j}\right| \quad i=1,2, \ldots, q
\end{aligned}
$$

Also, $a_{i i}$ can be replaced by Eq. (9) which gives

$$
\begin{gathered}
2 \sqrt{\tau^{n} \tau^{n+1}\left[4 m_{i i}^{2}-\left(\tau^{n} c_{i i}\right)^{2}\right.} \leq 2 m_{i i}\left(\tau^{n}+\tau^{n+1}\right)+\left(\tau^{n}-\tau^{n+1}\right) \tau^{n} c_{i i}-2\left(\tau^{n}\right)^{2} \tau^{n+1} S_{i i} \quad i=1,2, \ldots, q \\
2 \sqrt{\tau^{n} \tau^{n+1}\left[4 m_{i i}^{2}-\left(\tau^{n} c_{i i}\right)^{2}\right.} \leq 2\left(\tau^{n}\right)^{2} \tau^{n+1} \sum_{j=1}^{q}\left|S_{i j}\right|-2 m_{i i}\left(\tau^{n}+\tau^{n+1}\right)-\left(\tau^{n}-\tau^{n+1}\right) \tau^{n} c_{i i} \quad i=1,2, \ldots, q
\end{gathered}
$$

The fictitious parameters have real values if the expressions in the right side of inequalities (33) and (34) are greater than or equal to zero

$$
\begin{aligned}
& m_{i i} \geq \frac{\left(\tau^{n}\right)^{2} \tau^{n+1}}{\tau^{n}+\tau^{n+1}} S_{i i}-\frac{\tau^{n}-\tau^{n+1}}{\tau^{n}+\tau^{n+1}} \frac{\tau^{n} c_{i i}}{2} \quad i=1,2, \ldots, q \\
& m_{i i} \leq \frac{\left(\tau^{n}\right)^{2} \tau^{n+1}}{\tau^{n}+\tau^{n+1}} \sum_{j=1}^{q}\left|S_{i j}\right|-\frac{\tau^{n}-\tau^{n+1}}{\tau^{n}+\tau^{n+1}} \frac{\tau^{n} c_{i i}}{2} \quad i=1,2, \ldots, q
\end{aligned}
$$

Neglecting the variation of fictitious time step between two successive iterations leads to the simple relationship as follows

$$
\begin{gathered}
m_{i i} \geq \frac{\left(\tau^{n}\right)^{2}}{2} S_{i i} \quad i=1,2, \ldots, q \\
m_{i i} \leq \frac{\left(\tau^{n}\right)^{2}}{2} \sum_{j=1}^{q}\left|S_{i j}\right| \quad i=1,2, \ldots, q
\end{gathered}
$$


On the other hand, by combining Eqs. (15) and (16) one can write

$$
\lambda_{i U}^{B S}+\lambda_{i L}^{B S}=2\left(1+a_{i i}\right) \quad i=1,2, \ldots, q
$$

By considering Eqs. (18) and (39), it can be proved that

$$
2\left(1+a_{i i}\right) \geq \frac{2 \tau^{n+1} \tau^{n}}{2 m_{i i}+\tau^{n} c_{i i}} \sum_{j=1}^{q}\left|S_{i j}\right| \quad i=1,2, \ldots, q
$$

The above relationship, which has been proved in the appendix 1, leads to the following result

$$
m_{i i} \geq \frac{\left(\tau^{n}\right)^{2} \tau^{n+1}}{2\left(\tau^{n}+\tau^{n+1}\right)} \sum_{j=1}^{q}\left|S_{i j}\right|-\frac{\tau^{n}-\tau^{n+1}}{\tau^{n}+\tau^{n+1}} \frac{\tau^{n} c_{i i}}{2} \quad i=1,2, \ldots, q
$$

If the variation of time step between two successive iterations is neglected, simple relation is obtained

$$
m_{i i} \geq \frac{\left(\tau^{n}\right)^{2}}{4} \sum_{j=1}^{q}\left|S_{i j}\right| \quad i=1,2, \ldots, q
$$

As a result, the proposed formulation presents three inequalities for fictitious mass (Eqs. (35), (36) and (41) or Eqs. (37), (38) and 42). It is clear that fictitious mass is lied in the closed zone so that Eq. (36) shows the upper bound and Eqs. (35) and (41) denote the lower limits of this zone. As a result, it is necessary to introduce a reasonable approach for selection mass elements. In DR iterations, the residual force is one of the parameters which control the convergence. This quantity is composed from artificial inertia and damping forces of each iteration. By decreasing fictitious mass, which causes reduction in inertia forces, the residual force will reduce and one can expect that DR convergence rate will increase. The above discussion shows that the best and optimum selection for fictitious mass is the lower bound of the acceptable zone (Eqs. (35) and (41))

$$
\begin{gathered}
m_{i i}=\operatorname{MAX}\left[\frac{\left(\tau^{n}\right)^{2} \tau^{n+1}}{\tau^{n}+\tau^{n+1}} S_{i i}-\frac{\tau^{n}-\tau^{n+1}}{\tau^{n}+\tau^{n+1}} \frac{\tau^{n} c_{i i}}{2}, \frac{\left(\tau^{n}\right)^{2} \tau^{n+1}}{2\left(\tau^{n}+\tau^{n+1}\right)} \sum_{j=1}^{q}\left|S_{i j}\right|-\frac{\tau^{n}-\tau^{n+1}}{\tau^{n}+\tau^{n+1}} \frac{\tau^{n} c_{i i}}{2}\right] \\
i=1,2, \ldots, q
\end{gathered}
$$

If fictitious time step is assumed to be constant, simple relationship is obtained for fictitious mass entries

$$
m_{i i}=\operatorname{MAX}\left[\frac{\left(\tau^{n}\right)^{2}}{2} S_{i i}, \frac{\left(\tau^{n}\right)^{2}}{4} \sum_{j=1}^{q}\left|S_{i j}\right|\right] \quad i=1,2, \ldots, q
$$

It should be reminded that Eq. (42) is the most common relationship for calculating the fictitious mass in the ordinary DR algorithms. Therefore, the proposed formulation includes and embraces the previous methods. For protecting numerical instabilities in the ordinary Dynamic Relaxation method, the mass values from the Eq. (42) must be scaled by factor between 1.1 and 1.2. This scale factor should be selected by the analyst. Therefore, personal judgment has an effect on the ordinary DR algorithm. The presented formulation (Eq. (43) or (44)), solves this difficulty so that the proposed mass is found automatically.

Now, fictitious damping should be determined. It is clear that $[B]$ is a diagonal matrix. If Gerschgörin theory is used, the $i^{\text {th }}$ eigenvalue of $[B]^{-1}[S]$ can be assumed as follows 


$$
\lambda_{i}^{B S}=\frac{2 \tau^{n+1} \tau^{n}}{2 m_{i i}+\tau^{n} c_{i i}} \lambda_{i}^{S} \quad i=1,2, \ldots, q
$$

Here, $\lambda_{i}^{S}$ is the $\mathrm{i}^{\text {th }}$ eigenvalue of the stiffness matrix. The above assumption is logical because mass and damping are unknown parameters and they can be determined so that this assumption is valid (as done here). Substituting Eqs. (9) and (43) into the Eq. (13) leads to the second order equation

$$
Q_{i 1} c_{i i}^{2}+Q_{i 2} c_{i i}+Q_{i 3}=0 \quad i=1,2, \ldots, q
$$

In which

$$
\begin{gathered}
Q_{i 1}=\left(\tau^{n}\right)^{2}\left(\tau^{n}+\tau^{n+1}\right)^{2} \\
Q_{i 2}=4 m_{i i} \tau^{n}\left(\tau^{n}-\tau^{n+1}\right)\left[\tau^{n}+\tau^{n+1}-\left(\tau^{n}\right)^{2} \tau^{n+1} \omega_{i}^{2}\right] \\
Q_{i 3}=4 m_{i i}^{2}\left[\left(\tau^{n}-\tau^{n+1}\right)^{2}-2\left(\tau^{n}\right)^{2} \tau^{n+1}\left(\tau^{n}+\tau^{n+1}\right) \omega_{i}^{2}+\left(\tau^{n}\right)^{4}\left(\tau^{n+1}\right)^{2} \omega_{i}^{4}\right]
\end{gathered}
$$

Here, $\omega_{i}$ is the $\mathrm{i}^{\text {th }}$ natural frequency of the artificial dynamic system $\left(\omega_{i}^{2}=\lambda_{i}^{S} / m_{i i}\right)$. By assuming constant time step between two iterations, the following relationship is valid

$$
c_{i i}=\sqrt{\omega_{i}^{2}\left[4-\left(\tau^{n}\right)^{2} \omega_{i}^{2}\right]} m_{i i} \quad i=1,2, \ldots, q
$$

Analytical solution for calculating natural frequencies is complicated and time consuming procedure. Therefore, approximate technique can be used. In dynamic structural response, the effect of higher frequencies is less than the lower ones. Hence, the higher frequencies can be neglected and one can only uses the lowest fictitious frequency as

$$
c_{i i}=\sqrt{\omega_{\min }^{2}\left[4-\left(\tau^{n}\right)^{2} \omega_{\min }^{2}\right]} m_{i i} \quad i=1,2, \ldots, q
$$

The lowest frequency $\left(\omega_{\min }\right)$ of the structure can also be estimated from Rayleigh principle

$$
\omega_{\min }^{2}=\frac{\left(\{D\}^{n}\right)^{T}\{f\}^{n}}{\left(\{D\}^{n}\right)^{T}[M]^{n}\{D\}^{n}}
$$

In common DR methods, estimation of the critical damping is used for fictitious damping

$$
c_{i i}=2 \omega_{\min } m_{i i} \quad i=1,2, \ldots, q
$$

where $\omega_{\min }$ is calculated from Rayleigh principle (Eq. (52)).

\section{The proposed DR algorithms}

In the previous section, the error analysis was performed for DR iterations and new relationships for fictitious mass and damping were presented. First, general DR algorithm is given:

(a) Assume initial values for artificial velocity (null vector), displacement (null vector or convergence displacement on the previous increment, if available), fictitious time step $(\tau=1)$ and convergence criterion for out of balance force and kinetic energy $\left(e_{R}=1.0 \mathrm{E}-6\right.$ and $e_{K}=$ 
$1.0 \mathrm{E}-12)$.

(b) Construct tangent stiffness matrix and internal force vector.

(c) Apply boundary conditions.

(d) Calculate out of balance (residual) force vector using Eq. (5).

(e) If $\sqrt{\sum_{i=1}^{q}\left(r_{i}^{n}\right)^{2}} \leq e_{R}$, go to (1), otherwise, continue.

(f) Construct artificial diagonal mass matrix.

(g) Form artificial damping matrix.

(h) Update artificial velocity vector using Eq. (3).

(i) If $\sum_{i=1}^{q}\left(\dot{D}_{i}^{n+1 / 2}\right) \leq e_{K}$, go to (1), otherwise, continue.

(j) Update displacement vector using Eq. (4).

(k) Set $n=n+1$ and go to (b).

(l) Print results of the current increment.

(m) If increments are not complete, go to (a), otherwise, stop.

If increments are not complete, go to (a), otherwise, stop. If the proposed formulations are applied to the general DR algorithm, new method will be obtained. This method is called mdDR in which Eqs. (44) and (51) are used for fictitious mass and damping matrices, respectively. Also, fictitious damping should satisfy condition (14). For better studying the specification of the suggested formulation, the $\mathrm{mDR}$ algorithm is introduced so that fictitious mass and damping are calculated based on Eqs. (44) and (53), respectively. In the mDR algorithm, mass matrix is calculated from the proposed formulation but damping factor is calculated from the conventional method (Eq. (53)). Also, the oDR is ordinary Dynamic Relaxation algorithm in which fictitious mass and damping are calculated from Eqs. (42) and (53), respectively.

It should be noted that fictitious time step is usually assumed to be constant and equal to one. However, there are other approaches to calculate fictitious time step which can be used in any DR algorithm (Kadkhodayan et al. 2008). Studying the specifications of the suggested formulations for mass and damping is the goal of this paper. Hence, fictitious time step is kept constant in all of the mdDR, mDR and oDR algorithms.

\section{Numerical examples and discussion}

To investigate the capability of the proposed algorithms (mDR and mdDR) compared to the conventional scheme (oDR), some structure is analyzed from finite element and finite difference undergoing linear and elastic geometrical nonlinear behaviors. In order to solve these problems, a computer program has been written by the authors with fortran power station software.

\subsection{Truss spring system}

A nonlinear one degree of freedom system, which is shown in Fig. 1, will be analyzed. This structure is formed by a spring with stiffness $K_{S}=10.51 \mathrm{~N} / \mathrm{cm}$ and a truss element with axial 


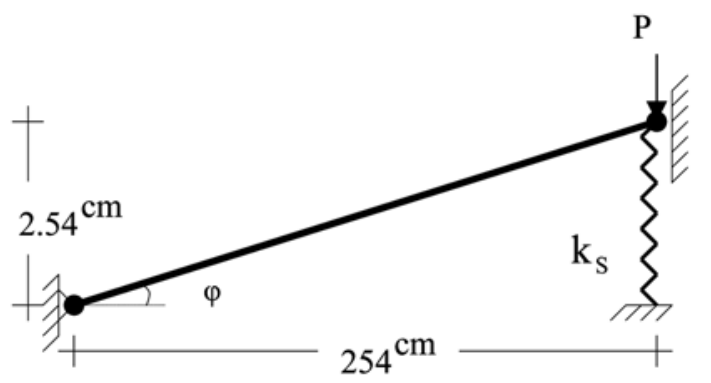

Fig. 1 Truss- spring system

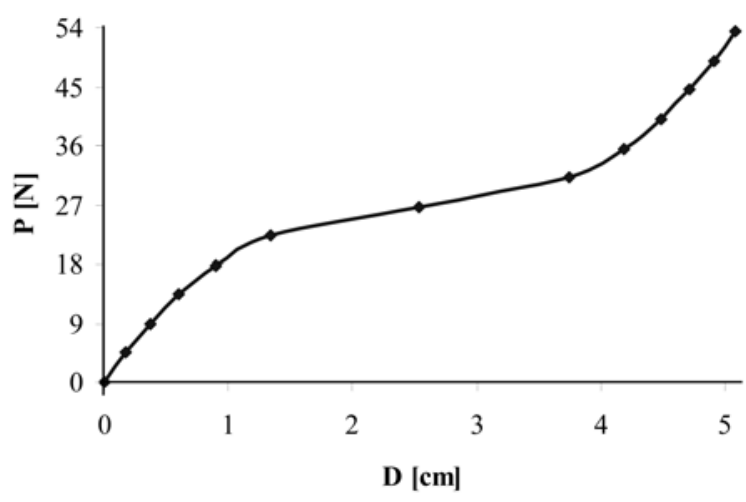

Fig. 2 Load-deflection curve for truss-spring system

Table 1 The number of iterations for convergence in the truss- spring system

\begin{tabular}{|c|c|c|c|c|c|c|c|c|c|c|c|c|c|c|c|}
\hline \multirow[b]{2}{*}{ Meth. } & \multicolumn{12}{|c|}{ Number of iterations for each load increment } & \multirow[b]{2}{*}{ Total } & \multicolumn{2}{|c|}{ Improvement $(\%)$} \\
\hline & 1 & 2 & 3 & 4 & 5 & 6 & 7 & 8 & 9 & 10 & 11 & 12 & & $\frac{\mathrm{oDR}-\mathrm{mDR}}{\mathrm{oDR}}$ & $\frac{\mathrm{oDR}-\mathrm{mdDR}}{\mathrm{oDR}}$ \\
\hline oDR & 76 & 73 & 77 & 83 & 95 & 134 & 75 & 57 & 49 & 45 & 42 & 40 & 846 & & \\
\hline $\mathrm{mDR}$ & 17 & 17 & 19 & 21 & 26 & 45 & 17 & 11 & 6 & 8 & 9 & 11 & 207 & 79.3 & 86.1 \\
\hline mdDR & 5 & 6 & 8 & 11 & 19 & 4 & 7 & 9 & 11 & 12 & 13 & 13 & 118 & & \\
\hline
\end{tabular}

rigidity of $\mathrm{AE}=44483985.77 \mathrm{~N}$. The fundamental relationships for internal force $(f)$ and tangent stiffness $\left(S_{T}\right)$ are as follows (Undewood 1983)

$$
\begin{gathered}
f(D)=0.5 A E\left(\operatorname{Cos}^{2} \varphi\right)\left(\frac{D}{L_{0}}\right)^{2}\left[\frac{D}{L_{0}} \operatorname{Cos}^{2} \varphi-3 \operatorname{Sin} \varphi\right]+k_{s} D+\left(A E \frac{D}{L_{0}}\right) \operatorname{Sin}^{2} \varphi \\
S_{T}=1.5 A E\left(\operatorname{Cos}^{2} \varphi\right)\left[\frac{D}{L_{0}} \operatorname{Cos}^{2} \varphi-2 \operatorname{Sin} \varphi\right]\left(\frac{D}{L_{0}^{2}}\right)+k_{s}+\frac{A E \operatorname{Sin}^{2} \varphi}{L_{0}}
\end{gathered}
$$

Loading process will be completed after twelve increments. The load growth is $4.4484 \mathrm{~N}$ in each increment. Fig. 2 displays the load-deflection curve for this system. The number of iterations for convergence, which has been inserted in Table 1 , shows that the mDR and mdDR algorithms cause maximum reduction up to about $79 \%$ and $86 \%$ compared to the oDR, respectively. Comparing the 


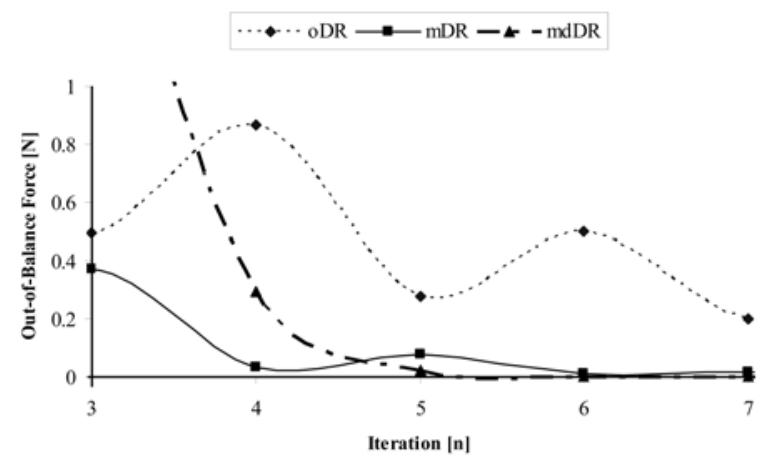

Fig. 3 Variation of out-of-balance force for the $7^{\text {th }}$ load increment of the truss-spring system

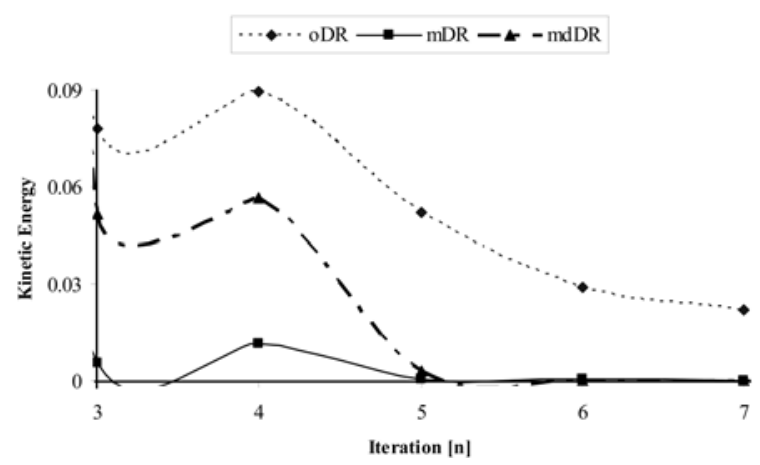

Fig. 4 Variation of kinetic energy for the $7^{\text {th }}$ load increment of the truss-spring system

results of the mDR and mdDR displays that the effect of the proposed mass on improving DR convergence rate is more than the effect of the suggested damping (Eq. (51)). For instance, the proposed mass and damping factors cause a reduction about $79 \%$ and $7 \%$ in DR iterations, respectively. On the other hand, Figs. 3 and 4 show variations of the out of balance force and kinetic energy for the $7^{\text {th }}$ increment, which has intense nonlinear behavior, respectively. From these figures, one can clearly see that the $\mathrm{mDR}$ and mdDR reduce the out-of-balance forces and the kinetic energy quicker than the oDR method.

\subsection{Toggle truss}

This single degree of freedom problem, shown in Fig. 5, is analyzed before its elastic buckling point (Ramesh and Krishnamoorthy 1994). The modulus of elasticity is $7.03 \mathrm{E} 5 \mathrm{~kg} / \mathrm{cm}^{2}$ and the section area of each element is $96.77 \mathrm{~cm}^{2}$. The ultimate value of external load is $3.2 \mathrm{E} 6 \mathrm{~kg}$. Linear and elastic geometrical nonlinear analyses are performed for ten loading increments. Total Lagrangian finite element approach is also utilized for elastic-large deformation formulation (Felippa 1999). Fig. 6 displays the load deflection curves of the vertical displacement of truss for both linear and nonlinear analyses. The number of iterations for convergence has also been inserted in Table 2. In linear analysis, the $\mathrm{mDR}$ and $\mathrm{mdDR}$ algorithms are converged to the solution only after one iteration. This means that the convergence rate of the suggested technique is infinite in linear behavior as it is proved mathematically in the appendix 2 . This property may also be

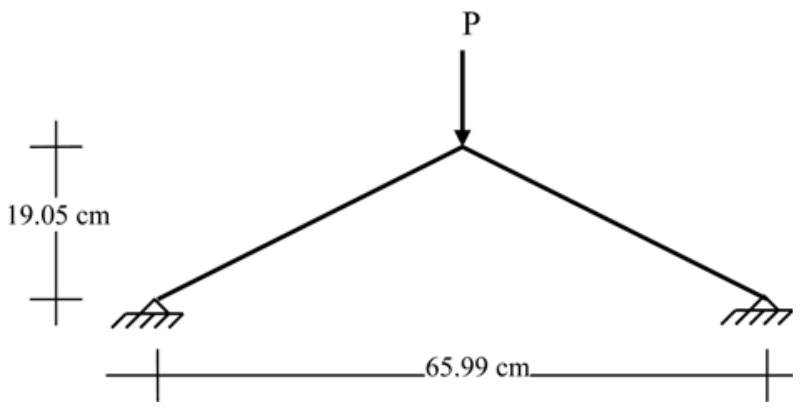

Fig. 5 The toggle truss 


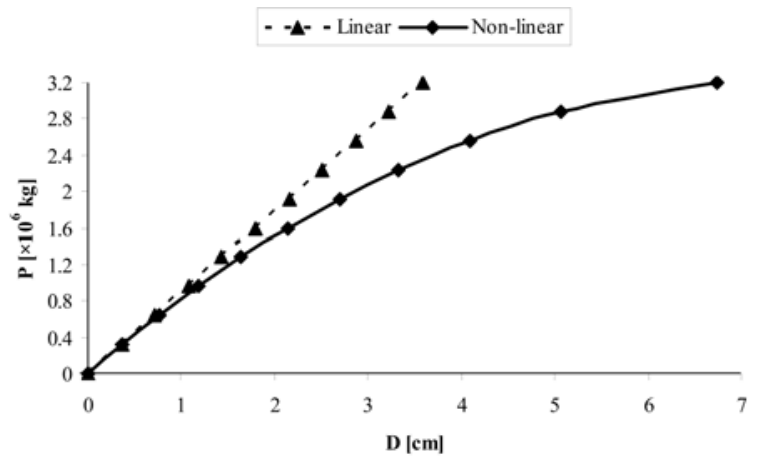

Fig. 6 Load-deflection curves for the toggle truss

Table 2 The number of iterations for convergence in the toggle truss

\begin{tabular}{|c|c|c|c|c|c|c|c|c|c|c|c|c|c|c|}
\hline \multirow[b]{2}{*}{ Analysis } & \multirow[b]{2}{*}{ Meth. } & \multicolumn{10}{|c|}{ Number of iterations for each load increment } & \multirow[b]{2}{*}{ Total } & \multicolumn{2}{|c|}{ Improvement (\%) } \\
\hline & & 1 & 2 & 3 & 4 & 5 & 6 & 7 & 8 & 9 & 10 & & $\frac{\mathrm{oDR}-\mathrm{mDR}}{\mathrm{oDR}}$ & $\frac{\mathrm{oDR}-\mathrm{mdDR}}{\mathrm{oDR}}$ \\
\hline \multirow{3}{*}{ Linear } & oDR & 144 & 132 & 132 & 132 & 132 & 132 & 132 & 132 & 132 & 132 & 1332 & \multirow{3}{*}{96.7} & \multirow{3}{*}{98.4} \\
\hline & $\mathrm{mDR}$ & 17 & 3 & 3 & 3 & 3 & 3 & 3 & 3 & 3 & 3 & 44 & & \\
\hline & mdDR & 3 & 2 & 2 & 2 & 2 & 2 & 2 & 2 & 2 & 2 & 21 & & \\
\hline \multirow{3}{*}{$\begin{array}{l}\text { Non- } \\
\text { linear }\end{array}$} & oDR & 146 & 137 & 140 & 144 & 149 & 155 & 163 & 176 & 198 & 279 & 1687 & \multirow{3}{*}{88.0} & \multirow{3}{*}{93.4} \\
\hline & $\mathrm{mDR}$ & 18 & 15 & 15 & 17 & 17 & 17 & 19 & 21 & 25 & 39 & 203 & & \\
\hline & mdDR & 6 & 6 & 7 & 7 & 9 & 9 & 11 & 15 & 37 & 5 & 112 & & \\
\hline
\end{tabular}
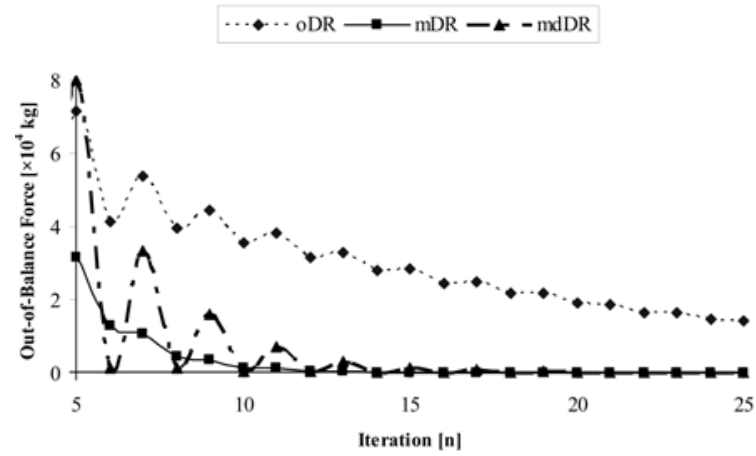

Fig. 7 Variation of out-of-balance force for the $9^{\text {th }}$ load increment of the toggle truss

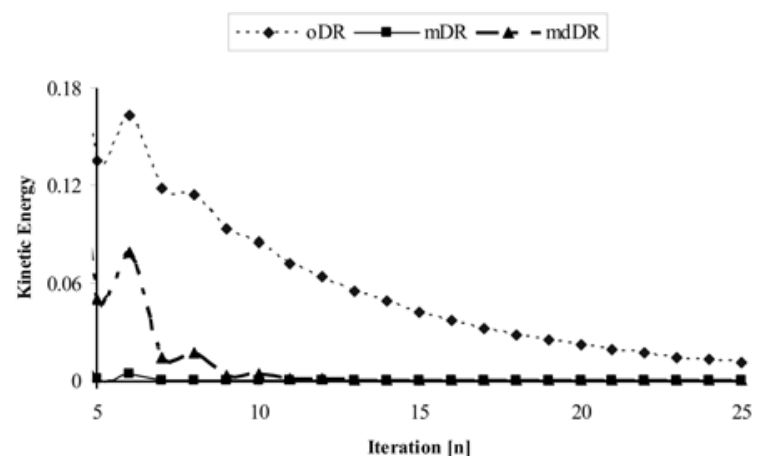

Fig. 8 Variation of kinetic energy for the $9^{\text {th }}$ load increment of the toggle truss

observed easily in other linear systems with single degree of freedom. By applying the mDR method, which uses only the proposed fictitious mass, maximum reduction up to $88 \%$ is achieved compared to the oDR. This improvement is up to about $94 \%$ if the mdDR algorithm is utilized. Comparing the results of the $\mathrm{mDR}$ and mdDR shows that the effect of the modified damping factor (Eq. (51)) which is about $6 \%$ is less than the effect of the proposed mass. Finally, the variations of un-balance force and kinetic energy have been plotted in Figs. 7 and 8 for the $9^{\text {th }}$ increment of 
nonlinear analyses, respectively. It is clear that the $\mathrm{mDR}$ and $\mathrm{mdDR}$ algorithms reduce the out-ofbalance forces along with the kinetic energy quicker than the oDR scheme.

\subsection{Space truss}

Fig. 9 shows a space truss with axial rigidity of $\mathrm{AE}=2.1 \mathrm{E} 6 \mathrm{KN}$. This structure has 21 nodes, 52 elements and 39 degrees of freedom (Ramesh and Krishnamoorthy 1994). The ultimate value of

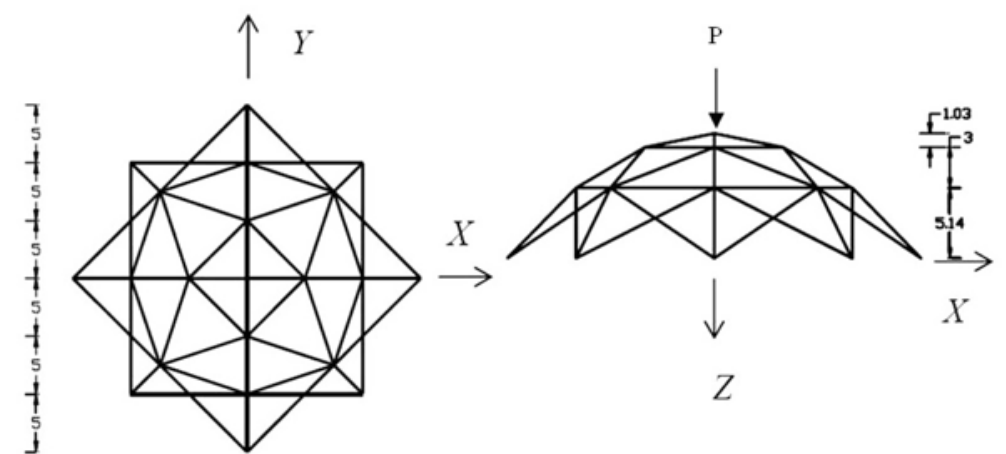

Fig. 9 The space truss (dimensions in meters and are the same for the $\mathrm{X}$ and $\mathrm{Y}$ axes)

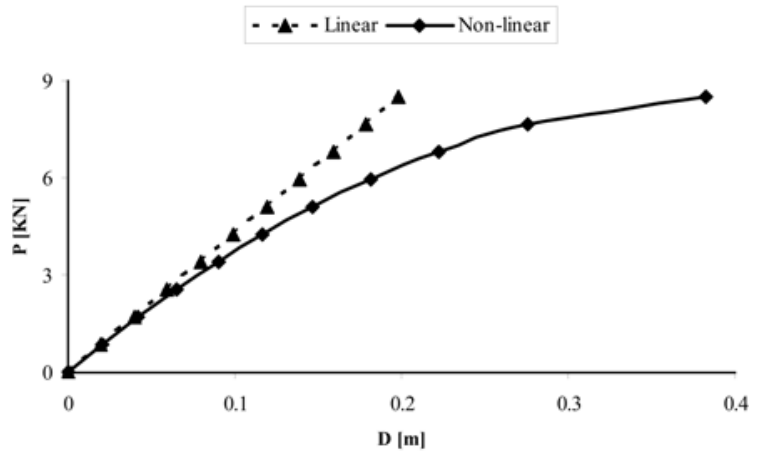

Fig. 10 Load-deflection curves for the space truss

Table 3 The number of iterations for convergence in the space truss

\begin{tabular}{|c|c|c|c|c|c|c|c|c|c|c|c|c|c|c|}
\hline \multirow[b]{2}{*}{ Analysis } & \multirow[b]{2}{*}{ Meth. } & \multicolumn{10}{|c|}{ Number of iterations for each load increment } & \multirow[b]{2}{*}{ Total } & \multicolumn{2}{|c|}{ Improvement (\%) } \\
\hline & & 1 & 2 & 3 & 4 & 5 & 6 & 7 & 8 & 9 & 10 & & $\frac{\mathrm{oDR}-\mathrm{mDR}}{\mathrm{oDR}}$ & $\frac{\mathrm{oDR}-\mathrm{mdDR}}{\mathrm{oDR}}$ \\
\hline \multirow{3}{*}{ Linear } & oDR & 41 & 40 & 40 & 40 & 40 & 40 & 40 & 40 & 40 & 40 & 401 & \multirow{3}{*}{4.7} & \multirow{3}{*}{7.7} \\
\hline & $\mathrm{mDR}$ & 39 & 39 & 38 & 38 & 38 & 38 & 38 & 38 & 38 & 38 & 382 & & \\
\hline & mdDR & 37 & 37 & 37 & 37 & 37 & 37 & 37 & 37 & 37 & 37 & 370 & & \\
\hline \multirow{3}{*}{$\begin{array}{l}\text { Non- } \\
\text { linear }\end{array}$} & oDR & 40 & 39 & 39 & 38 & 38 & 39 & 43 & 51 & 67 & 209 & 603 & \multirow{3}{*}{3.8} & \multirow{3}{*}{7.8} \\
\hline & $\mathrm{mDR}$ & 39 & 38 & 37 & 37 & 36 & 38 & 42 & 49 & 64 & 200 & 580 & & \\
\hline & mdDR & 37 & 36 & 36 & 35 & 35 & 36 & 40 & 47 & 61 & 193 & 556 & & \\
\hline
\end{tabular}




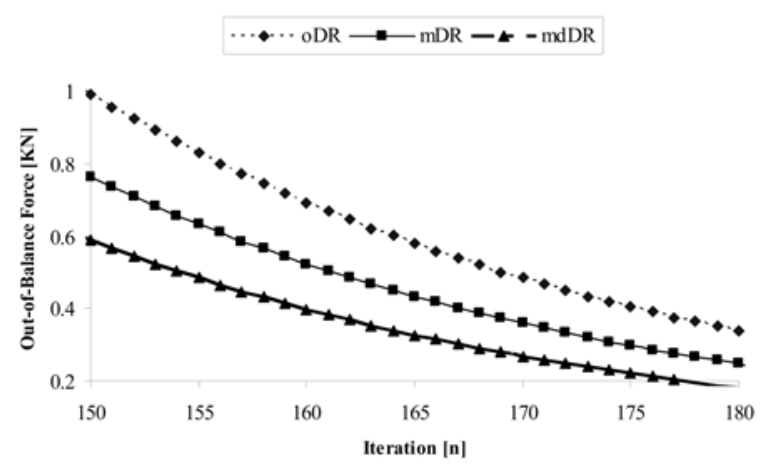

Fig. 11 Variation of out-of-balance force for the $10^{\text {th }}$ load increment of the space truss

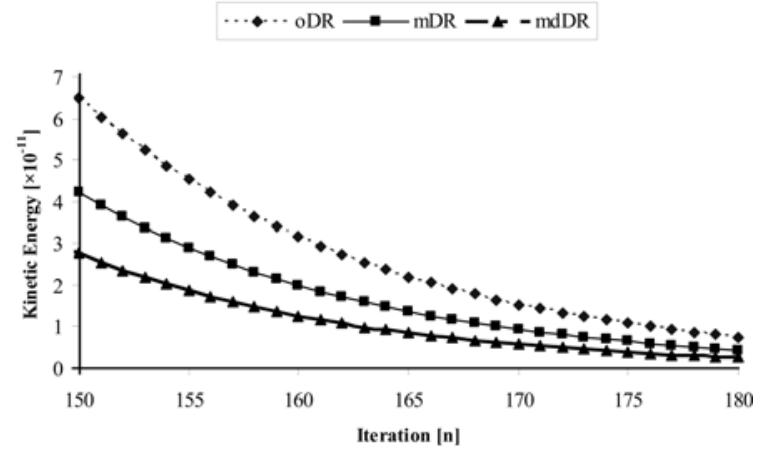

Fig. 12 Variation of kinetic energy for the $10^{\text {th }}$ load increment of the space truss

external load is $8500 \mathrm{KN}$ and the nodes in elevation $Z=0$ are restrained in all directions. Linear and elastic geometrical nonlinear analyses are performed for ten loading increments. Total Lagrangian finite element approach is utilized for elastic-large deformation formulation (Felippa 1999). Fig. 10 shows the load deflection curves for $Z$ direction displacement of the tip node in both linear and nonlinear analyses. The number of iterations for convergence has been inserted in Table 3 . When the mDR and mdDR are used, maximum reduction of iterations is up to about $4 \%$ and $7 \%$ compared to the oDR, respectively. Therefore, the proposed formulation for fictitious mass and damping factors has suitable effect on increasing the convergence rate of DR. For instance, the variations of un-balance force and kinetic energy have been plotted in Figs. 11 and 12 for the $10^{\text {th }}$ increment of nonlinear analysis, respectively. Because of the scale of values here that one may not be able to observe the differences between three algorithms clearly, when the total iterations are considered as a whole. Hence, the variations of un-balance force and kinetic energy have been plotted for a sample of iterations, i.e., iterations between 150 and 180; where the amounts of numerical values are closer to each other. These Figures display that the $\mathrm{mDR}$ and $\mathrm{mdDR}$ reduce the out-of-balance force and the kinetic energy quicker than when the ordinary dynamic relaxation algorithm is used.

\subsection{Building frame}

A building frame with five bays and six stories is shown in Fig. 13. A uniform load of $q=50 \mathrm{~kg} /$ $\mathrm{cm}$ is applied on each floor and the horizontal forces are calculated by distribution of the base shear resulting from earthquake loading. The columns of three downer and upper stories are constructed by $\mathrm{W} 18 \times 40$ and $\mathrm{W} 18 \times 35$, respectively. Also, all beams are $\mathrm{W} 16 \times 31$. Small and elastic-large deformation analyses will be performed for this structure. The co-rotational finite element formulation is also utilized for large-deformation analysis (Saka 1990). Fig. 14 shows the loaddeflection curves of the horizontal displacement of the top of fame for both linear and nonlinear analyses. The number of iterations for convergence has also been inserted in Table 4. Here, both the $\mathrm{mDR}$ and $\mathrm{mdDR}$ procedures cause average reduction about $4 \%$ in required iterations compared to the oDR method. In other words, the results of the mDR and mdDR are the same. Therefore, the proposed damping factor (Eq. (51)) does not improve the convergence rate of DR and does not have any suitable effect. The reason for this property can be illustrated as follows. In such great structure, 


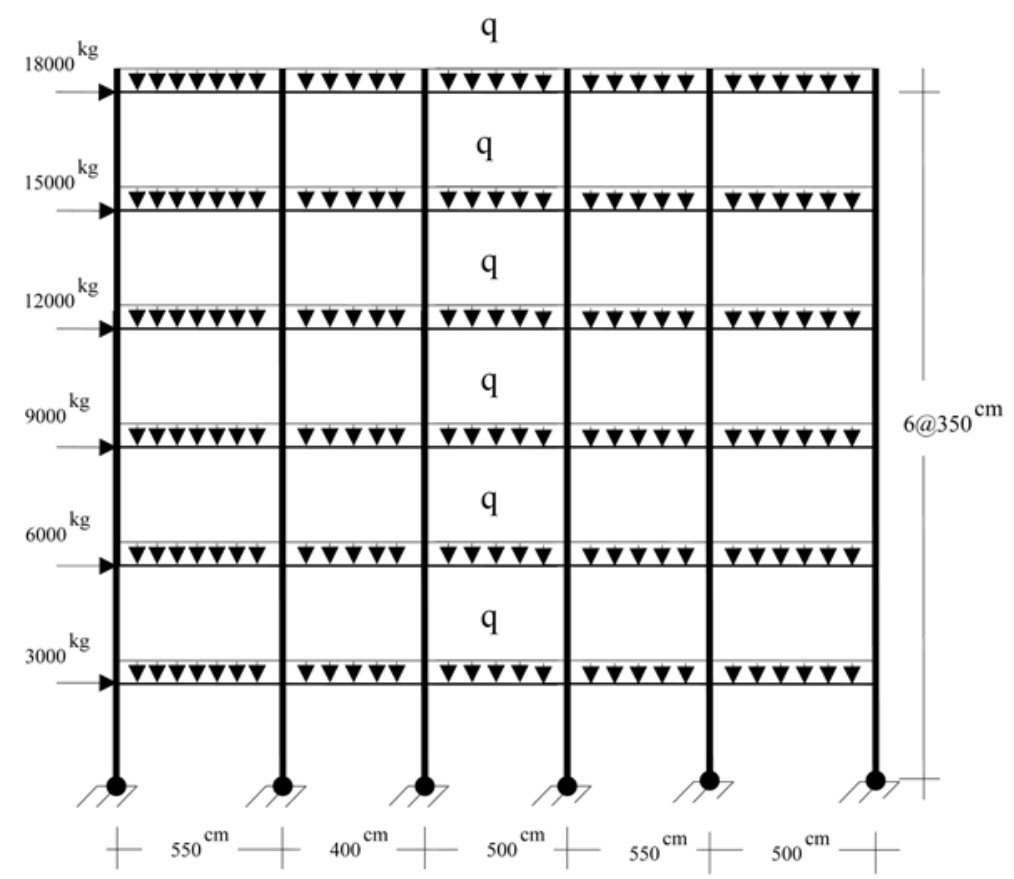

Fig. 13 The building frame

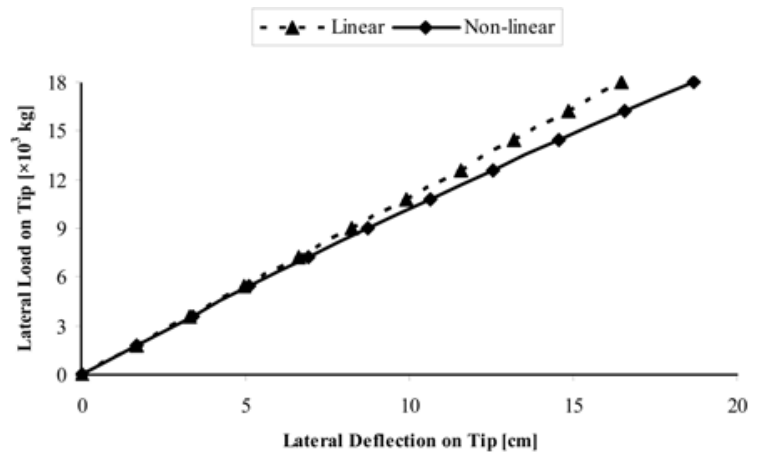

Fig. 14 Load-deflection curves for the building frame

Table 4 The number of iterations for convergence in the building frame

\begin{tabular}{|c|c|c|c|c|c|c|c|c|c|c|c|c|c|c|}
\hline \multirow[b]{2}{*}{ Analysis } & \multirow[b]{2}{*}{ Meth. } & \multicolumn{10}{|c|}{ Number of iterations for each load increment } & \multirow[b]{2}{*}{ Total } & \multicolumn{2}{|c|}{ Improvement (\%) } \\
\hline & & 1 & 2 & 3 & 4 & 5 & 6 & 7 & 8 & 9 & 10 & & $\frac{\mathrm{oDR}-\mathrm{mDR}}{\mathrm{oDR}}$ & $\frac{\mathrm{oDR}-\mathrm{mdDR}}{\mathrm{oDR}}$ \\
\hline \multirow{3}{*}{ Linear } & oDR & 2090 & 1626 & 1585 & 1568 & 1559 & 1554 & 1550 & 1547 & 1545 & 1543 & 16167 & \multirow{3}{*}{4.1} & \multirow{3}{*}{4.1} \\
\hline & $\mathrm{mDR}$ & 2002 & 1560 & 1521 & 1505 & 1496 & 1491 & 1487 & 1485 & 1483 & 1481 & 15511 & & \\
\hline & mdDR & 2001 & 1560 & 1520 & 1505 & 1496 & 1491 & 1487 & 1485 & 1483 & 1481 & 15509 & & \\
\hline \multirow{3}{*}{$\begin{array}{l}\text { Non- } \\
\text { linear }\end{array}$} & oDR & 2102 & 1651 & 1623 & 1621 & 1627 & 1637 & 1650 & 1664 & 1679 & 1696 & 16950 & \multirow{3}{*}{4.1} & \multirow{3}{*}{4.1} \\
\hline & $\mathrm{mDR}$ & 2013 & 1584 & 1558 & 1556 & 1562 & 1571 & 1583 & 1597 & 1612 & 1628 & 16264 & & \\
\hline & mdDR & 2012 & 1583 & 1558 & 1556 & 1562 & 1571 & 1583 & 1597 & 1612 & 1628 & 16262 & & \\
\hline
\end{tabular}




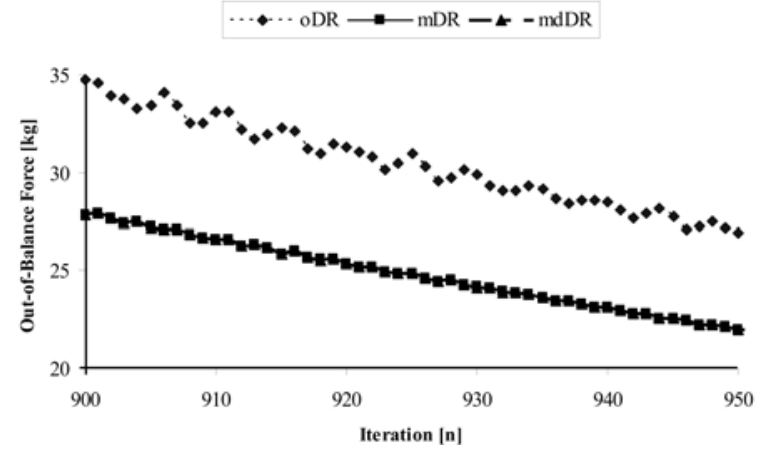

Fig. 15 Variation of out-of-balance force for the $10^{\text {th }}$ load increment of the building frame

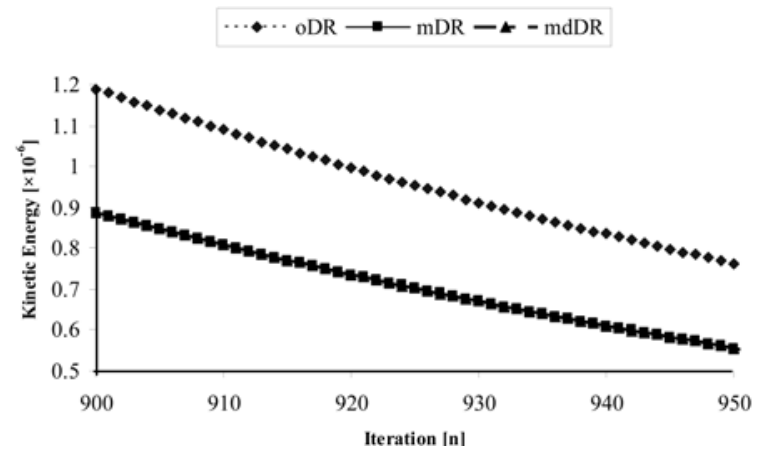

Fig. 16 Variation of kinetic energy for the $10^{\text {th }}$ load increment of the building frame

with 114 degrees of freedom, the lowest fictitious natural frequency $\left(\omega_{\min }\right)$ is near zero. Therefore, the effect of $\omega_{\min }^{2}$ compared to $\omega_{\min }$ can be neglected. As a result, Eq. (51) is transformed to Eq. (53). Similar behavior can be expected in other multi degrees of freedom systems. However, the proposed mass formulation still has a considerable effect on improving DR convergence rate.

It should be noted that calculating of the internal force vector and stiffness matrix are very expensive in nonlinear analyses. Hence, reduction of number of iterations decreases the cost and the computational time considerably. As a result, using the suggested formulation for fictitious mass is strongly advised for nonlinear multi degrees of freedom structures, such as frames. Although, in nonlinear problems, mathematical convergence rank of the oDR and mdDR are the same and equal to one but, the numerical results show that the proposed method requires less iterations than the ordinary dynamic relaxation technique. Therefore, the convergence rate of the mdDR is higher than the conventional DR algorithm. For instance, the variations of un-balance force and kinetic energy have been illustrated by Figs. 15 and 16 for the $10^{\text {th }}$ increment of nonlinear analyses, respectively. Because of the scale of values, one may not be able to observe the differences between the mDR, mdDR and oDR clearly, when the total iterations are considered as a whole. Hence, the variations of un-balance force and kinetic energy have been plotted for iterations between 900 and 950 of nonlinear analysis. In this range, the amounts of numerical values are closer to each other. These Figures display that the proposed algorithms (mDR and mdDR) reduces the out-of-balance force and the kinetic energy quicker than the oDR algorithm.

\subsection{Plate structure}

The incremental form of the plate equilibrium equations may be written in the following form (Kadkhodayan et al. 1997)

$$
\begin{aligned}
& \frac{\partial \delta N_{x}}{\partial x}+\frac{\partial \delta N_{x y}}{\partial y}=0 \\
& \frac{\partial \delta N_{y}}{\partial y}+\frac{\partial \delta N_{x y}}{\partial x}=0
\end{aligned}
$$




$$
\begin{gathered}
\frac{\partial^{2} \delta M_{x}}{\partial x^{2}}+\frac{\partial^{2} \delta M_{y}}{\partial y^{2}}-2 \frac{\partial^{2} \delta M_{x y}}{\partial x \partial y}+\delta N_{x} \frac{\partial^{2} w}{\partial x^{2}}+\left(N_{x}+\delta N_{x}\right) \frac{\partial^{2} \delta w}{\partial x^{2}} \\
+\delta N_{y} \frac{\partial^{2} w}{\partial y^{2}}+\left(N_{y}+\delta N_{y}\right) \frac{\partial^{2} \delta w}{\partial y^{2}}+2 \delta N_{x y} \frac{\partial^{2} w}{\partial x \partial y}+2\left(N_{x y}+\delta N_{x y}\right) \frac{\partial^{2} \delta w}{\partial x \partial y}+\delta q=0
\end{gathered}
$$

In Eqs. (56)-(58) the symbol $\delta$ denotes the incremental change in quantities compared to their converged magnitude at the end of the previous load step. The incremental membrane forces and bending moments may be expressed as

$$
\left(\delta N_{x}, \delta N_{y}, \delta N_{x y}, \delta M_{x}, \delta M_{y}, \delta M_{x y}\right)=\int_{-h / 2}^{h / 2}\left(\delta \sigma_{x}, \delta \sigma_{y}, \delta \tau_{x y}, z \delta \sigma_{x}, z \delta \sigma_{y}, z \delta \tau_{x y}\right) d z
$$

where the incremental stresses are as below (Ugural 1987)

$$
\begin{gathered}
\left\{\begin{array}{l}
\delta \sigma_{x} \\
\delta \sigma_{y} \\
\delta \tau_{x y}
\end{array}\right\}=G\left\{\begin{array}{c}
2 \delta \varepsilon_{x} \\
2 \delta \varepsilon_{y} \\
\delta \gamma_{x y}
\end{array}\right\}+\lambda e\left\{\begin{array}{l}
1 \\
1 \\
0
\end{array}\right\} \\
G=\frac{E}{2(1+v)}, \quad e=\delta \varepsilon_{x}+\delta \varepsilon_{y}, \quad \lambda=\frac{v E}{(1+v)(1-2 v)}
\end{gathered}
$$

where $E, G, v, \lambda$ and $e$ are the Young's modulus, shear modulus, Poisson's ratio, Lame' coefficient and volumetric strain of the material, respectively. The incremental strains of points at a distance $z$ from the middle plane of the plate are given by

$$
\left\{\begin{array}{l}
\delta \varepsilon_{x} \\
\delta \varepsilon_{y} \\
\delta \gamma_{x y}
\end{array}\right\}=\left\{\begin{array}{l}
\delta \varepsilon_{x}^{0} \\
\delta \varepsilon_{y}^{0} \\
\delta \gamma_{x y}^{0}
\end{array}\right\}+z\left\{\begin{array}{l}
\delta \kappa_{x} \\
\delta \kappa_{y} \\
\delta \kappa_{x y}
\end{array}\right\}
$$

The incremental mid-plane normal and shear strains and curvatures of plate are given below (Ugural 1987)

$$
\begin{gathered}
\delta \varepsilon_{x}^{0}=\frac{\partial \delta u}{\partial x}+\frac{1}{2}\left(\frac{\partial \delta w}{\partial x}\right)^{2}+\frac{\partial w}{\partial x} \frac{\partial \delta w}{\partial x} \\
\delta \varepsilon_{y}^{0}=\frac{\partial \delta v}{\partial y}+\frac{1}{2}\left(\frac{\partial \delta w}{\partial y}\right)^{2}+\frac{\partial w}{\partial y} \frac{\partial \delta w}{\partial y} \\
\delta \gamma_{x}^{0}=\frac{\partial \delta u}{\partial y}+\frac{\partial \delta v}{\partial x}+\frac{\partial w}{\partial x} \frac{\partial w}{\partial y}+\frac{\partial w}{\partial x} \frac{\partial \delta w}{\partial y}+\frac{\partial \delta w}{\partial x} \frac{\partial w}{\partial y}+\frac{\partial \delta w}{\partial x} \frac{\partial \delta w}{\partial y} \\
\delta \kappa_{x}=-\frac{\partial^{2} \delta w}{\partial x^{2}} \\
\delta \kappa_{y}=-\frac{\partial^{2} \delta w}{\partial y^{2}} \\
\delta \kappa_{x y}=2 \frac{\partial^{2} \delta w}{\partial x \partial y}
\end{gathered}
$$


where $\delta u, \delta v$ and $\delta w$ ware the incremental displacement components in the $x, y$ and $z$ directions, respectively.

In order to compute the numerical derivatives of the incremental bending and twisting moments $\left(\delta M_{x}, \delta M_{y}, \delta M_{x y}\right)$, lateral displacement $(\delta w)$, membrane forces $\left(\delta N_{x}, \delta N_{y}, \delta N_{x y}\right)$ and in-plane displacements $(\delta u, \delta v)$ central, forward and backward finite difference (FD) schemes are used, as appropriate. After applying the boundary conditions, all quantities are updated by substituting the increments into the FD forms of the differential equation for each node.

In the case of small deflection bending theory, the effects of membrane forces and in-plane displacements are negligible. Hence, the strains in the mid-plane of the plate are ignored $\left(\delta \varepsilon_{x}^{0}=\delta \varepsilon_{y}^{0}\right.$ $\left.=\delta \gamma_{x y}^{0}=0\right)$. Therefore, there is only one plate equilibrium equation

$$
\frac{\partial^{2} \delta M_{x}}{\partial x^{2}}+\frac{\partial^{2} \delta M_{y}}{\partial y^{2}}-2 \frac{\partial^{2} \delta M_{x y}}{\partial x \partial y}+\delta q=0
$$

The other equations can be re-arranged accordingly.

The plate deformation is analyzed using both small and large deflection plate equations. The Young's modulus $(E)$ and Poisson's ratio $(v)$ of the plate material are taken as $210 \mathrm{KN} / \mathrm{mm}^{2}$ and 0.3 , respectively. The plate has an aspect ratio $(a / b)$ of 1 and a width to thickness ratio $(a / h)$ of 100 and it is subjected to a uniform transverse pressure.

In order to verify the accuracy of the method, the plate is assumed to be rectangular, so that $c_{1}=c_{2}=d_{1}=d_{2}=0$ in Fig. 17. Table 5 shows a comparison between the numerical and analytical results (Timoshenko and Woinowsky-Krieger 1959) for plates with different boundary conditions. The symbols $\mathrm{S}, \mathrm{C}$ and $\mathrm{F}$ in the table refer to simply supported, clamped and free edges and S.D. and L.D. refer to the small and large deflection plate theories, respectively (Timoshenko and Woinowsky-Krieger 1959). It is clear that there is a good agreement between the numerical and analytical results.

The consequences of applying the proposed algorithms will now be addressed using a specific

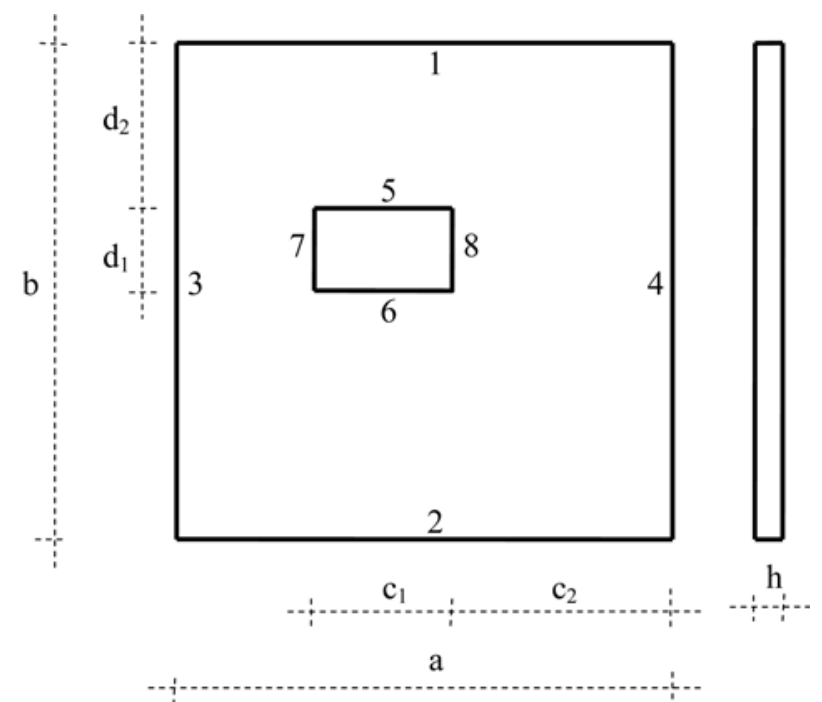

Fig. 17 Plan view of a punched plate with edges labelled 1-8 [Note: The plate reduces to a rectangular plate when $\left.c_{1}=c_{2}=d_{1}=d_{2}=0\right]$ 
Table 5 Comparison between numerical and analytical results for a uniformly loaded square plate $\left(c_{1}=c_{2}=\right.$

\begin{tabular}{|c|c|c|c|c|c|c|c|c|c|}
\hline \multirow[t]{2}{*}{ Case } & \multicolumn{4}{|c|}{$\begin{array}{l}\text { Boundary conditions } \\
\text { along edges }\end{array}$} & \multirow{2}{*}{$\begin{array}{l}\text { Deformation } \\
\text { type }\end{array}$} & \multirow{2}{*}{$\frac{12 q b^{4}\left(1-v^{2}\right)}{E h^{4}}$} & \multicolumn{2}{|c|}{$\frac{w_{\max }}{h}$} & \multirow{2}{*}{ 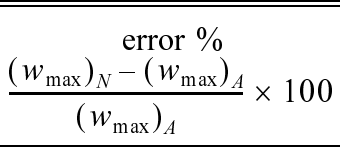 } \\
\hline & 1 & 2 & 3 & 4 & & & Analytical & Numerical & \\
\hline S1 & $\mathrm{C}$ & $\mathrm{C}$ & $\mathrm{C}$ & $\mathrm{C}$ & L.D.* & 1000 & 0.88 & 0.8971 & +1.9 \\
\hline $\mathrm{S} 2$ & $\mathrm{C}$ & $\mathrm{C}$ & $\mathrm{C}$ & $\mathrm{C}$ & S.D.** & 1000 & 1.25 & 1.2632 & +1.1 \\
\hline $\mathrm{S} 3$ & $\mathrm{~S}$ & $\mathrm{~S}$ & $\mathrm{C}$ & $\mathrm{C}$ & L.D. & 500 & 0.68 & 0.7005 & +3.1 \\
\hline S4 & $\mathrm{S}$ & $\mathrm{S}$ & $\mathrm{C}$ & $\mathrm{F}$ & S.D. & 100 & 0.83 & 0.8064 & -2.8 \\
\hline S5 & $\mathrm{S}$ & $\mathrm{S}$ & $\mathrm{S}$ & $\mathrm{F}$ & S.D. & 100 & 0.89 & 0.9123 & 2.5 \\
\hline
\end{tabular}

*Large Deformation

**Small Deformation

Table 6 The numbers of iterations for convergence in the DR finite difference analysis of punched plates for small deflection

\begin{tabular}{|c|c|c|c|c|c|c|c|c|c|c|c|c|c|c|c|}
\hline \multirow{2}{*}{ Case } & \multicolumn{8}{|c|}{ Boundary conditions along edges } & \multirow{2}{*}{$-\frac{12 q b^{4}\left(1-v^{2}\right)}{E h^{4}}$} & \multirow{2}{*}{$\frac{w_{\max }}{h}$} & \multicolumn{3}{|c|}{ Number of iterations } & \multicolumn{2}{|c|}{ Improvement (\%) } \\
\hline & 1 & 2 & 3 & 4 & 5 & 6 & 7 & 8 & & & Odr & $\mathrm{mDR}$ & mdDR & $\frac{\mathrm{oDR}-\mathrm{mDR}}{\mathrm{oDR}}$ & $\frac{\mathrm{oDR}-\mathrm{mdDR}}{\mathrm{oDR}}$ \\
\hline P1 & $\mathrm{S}$ & $\mathrm{S}$ & $\mathrm{C}$ & $\mathrm{C}$ & $\mathrm{S}$ & $\mathrm{S}$ & $\mathrm{S}$ & $\mathrm{S}$ & 2000 & 0.4749 & 424 & 405 & 405 & 4.5 & 4.5 \\
\hline $\mathrm{P} 2$ & $\mathrm{~S}$ & $\mathrm{~S}$ & $\mathrm{~S}$ & $\mathrm{~S}$ & $\mathrm{~S}$ & $\mathrm{~S}$ & $\mathrm{~S}$ & $\mathrm{~S}$ & 2000 & 0.5132 & 441 & 421 & 421 & 4.5 & 4.5 \\
\hline P3 & $\mathrm{F}$ & $\mathrm{C}$ & $\mathrm{S}$ & $\mathrm{S}$ & $\mathrm{C}$ & $\mathrm{C}$ & $\mathrm{S}$ & $\mathrm{S}$ & 500 & 0.7217 & 731 & 697 & 697 & 4.7 & 4.7 \\
\hline P4 & $\mathrm{F}$ & $\mathrm{C}$ & $\mathrm{C}$ & $\mathrm{C}$ & $\mathrm{S}$ & $\mathrm{S}$ & $\mathrm{S}$ & $\mathrm{F}$ & 500 & 0.8731 & 774 & 737 & 737 & 4.8 & 4.8 \\
\hline P5 & F & $\mathrm{C}$ & $\mathrm{S}$ & $\mathrm{C}$ & $\mathrm{C}$ & $\mathrm{F}$ & $\mathrm{S}$ & $\mathrm{S}$ & 500 & 0.6316 & 732 & 698 & 698 & 4.6 & 4.6 \\
\hline
\end{tabular}

Table 7 The numbers of iterations for convergence in the DR finite difference analysis of punched plates for large deflection

\begin{tabular}{|c|c|c|c|c|c|c|c|c|c|c|c|c|c|c|c|}
\hline \multirow[b]{2}{*}{ Case } & \multicolumn{8}{|c|}{ Boundary conditions along edges } & \multirow{2}{*}{$\frac{12 q b^{4}\left(1-v^{2}\right)}{E h^{4}}$} & \multirow[b]{2}{*}{$\frac{w_{\max }}{h}$} & \multicolumn{3}{|c|}{ Number of iterations } & \multicolumn{2}{|c|}{ Improvement (\%) } \\
\hline & 1 & 2 & 3 & 4 & 5 & 6 & 7 & 8 & & & oDR & $\mathrm{mDR}$ & mdDR & $\frac{\mathrm{oDR}-\mathrm{mDR}}{\mathrm{oDR}}$ & $\frac{\mathrm{oDR}-\mathrm{mdDR}}{\mathrm{oDR}}$ \\
\hline $\mathrm{P} 1$ & $\mathrm{~S}$ & $\mathrm{~S}$ & $\mathrm{C}$ & $\mathrm{C}$ & $\mathrm{S}$ & $\mathrm{S}$ & $\mathrm{S}$ & $\mathrm{S}$ & 2000 & 0.3832 & 243 & 233 & 233 & 4.1 & 4.1 \\
\hline $\mathrm{P} 2$ & $\mathrm{~S}$ & $\mathrm{~S}$ & $\mathrm{~S}$ & $\mathrm{~S}$ & $\mathrm{~S}$ & $\mathrm{~S}$ & $\mathrm{~S}$ & $\mathrm{~S}$ & 2000 & 0.3950 & 253 & 242 & 242 & 4.3 & 4.3 \\
\hline P3 & $\mathrm{F}$ & $\mathrm{C}$ & $\mathrm{S}$ & $\mathrm{S}$ & $\mathrm{C}$ & $\mathrm{C}$ & $\mathrm{S}$ & $\mathrm{S}$ & 500 & 0.7553 & 1129 & 1071 & 1072 & 5.1 & 5.0 \\
\hline P4 & $\mathrm{F}$ & $\mathrm{C}$ & $\mathrm{C}$ & $\mathrm{C}$ & $\mathrm{S}$ & $\mathrm{S}$ & $\mathrm{S}$ & $\mathrm{F}$ & 500 & 0.8785 & 1075 & 1019 & 1020 & 5.2 & 5.1 \\
\hline P5 & $\mathrm{F}$ & $\mathrm{C}$ & $\mathrm{S}$ & $\mathrm{C}$ & $\mathrm{C}$ & $\mathrm{F}$ & $\mathrm{S}$ & $\mathrm{S}$ & 500 & 0.6608 & 1122 & 1063 & 1064 & 5.3 & 5.2 \\
\hline
\end{tabular}

example. A punched plate with following geometry: $a / b=1, c_{1} / a=0.4, c_{2} / a=0.3, d_{1} / b=0.25, d_{2} /$ $b=0.35$ and $a / h=100$ is selected for this purpose. In this example, different plate theories and boundary conditions are also applied. The number of mesh intervals used in the analyses was 20, 20 and 9 for the length, width and thickness directions, respectively. The results obtained are shown in Tables 6 and 7 for small and large deflection, respectively. They indicate that when the mDR and mdDR algorithms are used, the average reduction in iterations for small and large deflection theory 


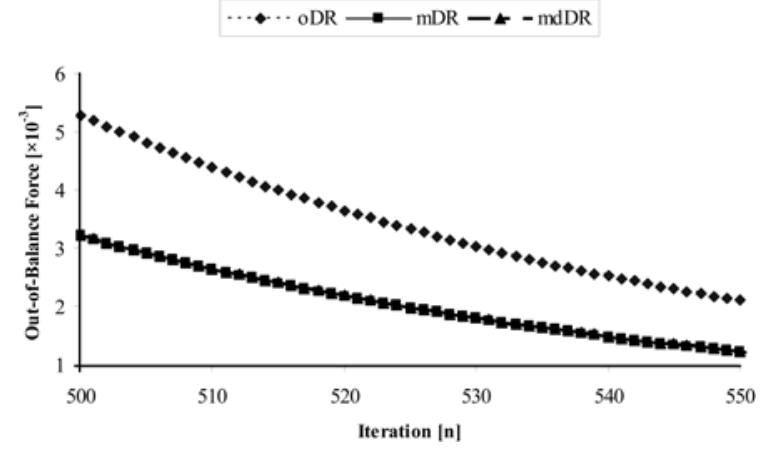

Fig. 18 Variation of out-of-balance force for small deflection of the case P5

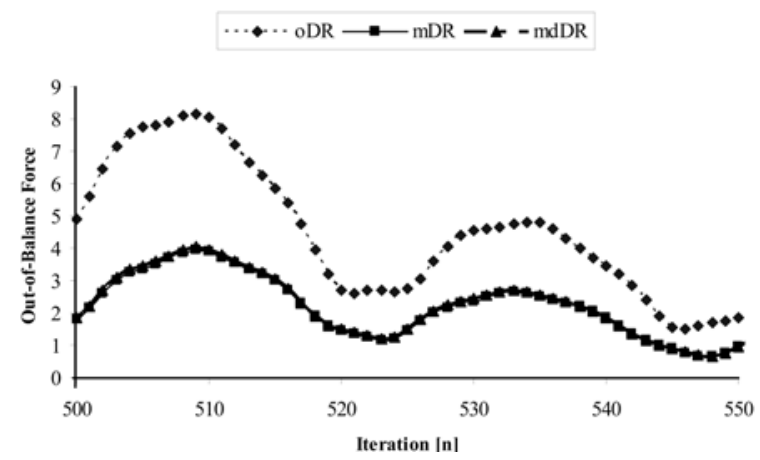

Fig. 20 Variation of out-of-balance force for large deflection of the case P5

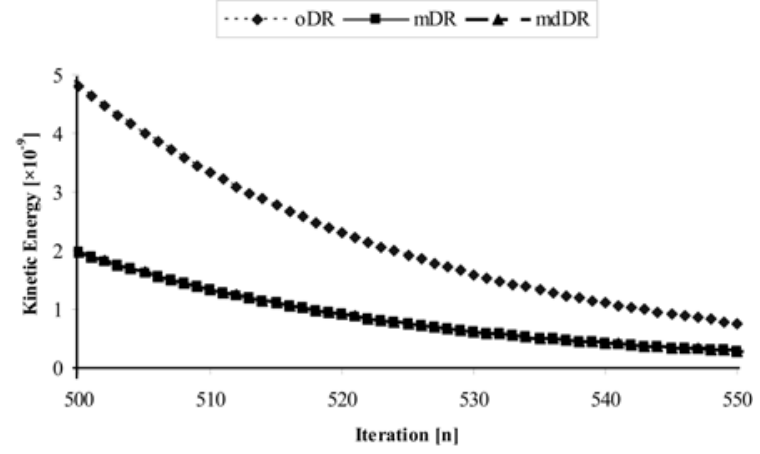

Fig. 19 Variation of kinetic energy for small deflection of the case P5

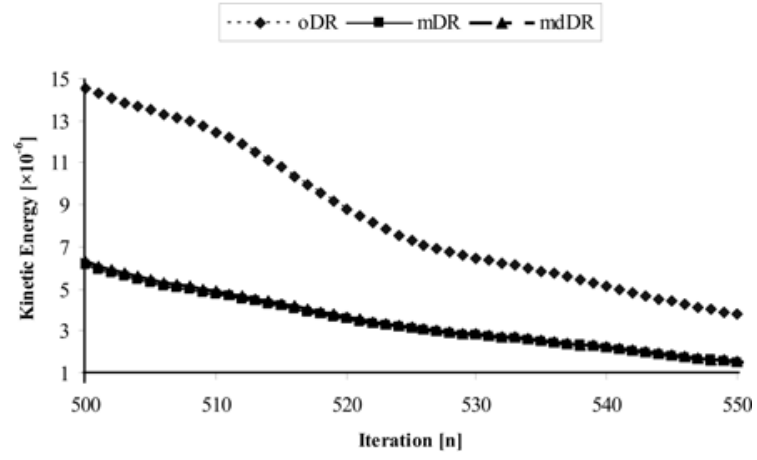

Fig. 21 Variation of kinetic energy for large deflection of the case P5

is about $5 \%$ compared to the ordinary DR method. It is clear that the number of iterations for convergence in the $\mathrm{mDR}$ and mdDR schemes is the same because, there is up to 300 degrees of freedom in the punched plate and its lowest fictitious natural frequency is near zero. Hence, the effect of the proposed damping factor is not considerable (as seen in Tables 6 and 7). On the other hand, the proposed fictitious mass still increases the convergence rate of DR method in finite difference analysis, in which numerical techniques are utilized. Additional quantitative evidence of the beneficial effect of the proposed formulation on the convergence rate of DR method may be seen by monitoring changes in the out-of-balance forces and the kinetic energy as the iterations progress. Plots of these quantities show the rate at which the dynamic response diminishes as the static solution is approached. As illustrative examples, plots the out-of-balance forces and kinetic energy have been created for plates with different types of boundary conditions, i.e., case P5. They are shown in Figs. 18-21 for both small and large deflection. Because of the scale of values, the differences between the out-of balance forces and kinetic energy are shown only for the iterations between 500 and 550. From these figures, it is evident that the mDR and mdDR procedures reduce the out-of-balance forces and the kinetic energy quicker than when the ordinary dynamic relaxation algorithm is used. 


\section{Conclusions}

In this paper, new formulation for fictitious mass and damping of Dynamic Relaxation method was introduced by minimizing error between two successive iterations. In the ordinary method personal judgment has an important effect on the selection of fictitious mass (scale factor of Eq. (42)) which affect the stability and efficiency of DR iterations. But, the suggested technique calculates fictitious mass automatically without any additional calculations. Moreover and for linear systems with one degree of freedom, using the proposed algorithm (mdDR) improves the convergence rank from one to infinity. The effects of proposed method were investigated for both finite difference (small and large deflection of plate bending problems with different boundary conditions) and finite element (linear and non-linear truss and frame structures with few and many degrees of freedom) problems. It is concluded that the number of iterations for convergence may be reduced by up to about $80 \%$ and $5 \%$ for systems with few and many degrees of freedom, respectively. Moreover, in great structures in which the lowest fictitious natural frequency is near zero, the proposed damping factor is transformed to the conventional damping. It should be mentioned that the suggested formulation does not impose any additional calculations.

\section{References}

Alwar, R.S., Rao, N.R. and Rao, M.S. (1975), “Altenative procedure in dynamic relaxation”, Comput. Struct., 5, 271-274.

Bardet, J.P. and Proubet, J. (1991), "Adaptive dynamic relaxation for statics of granular materials", Comput. Struct., 39, 221-229.

Brew, J.S. and Brotton, M. (1971), "Non-linear structural analysis by dynamic relaxation method", Int. J. Numer. Meth. Eng., 3, 463-483.

Bunce, J.W. (1972), "A note on estimation of critical damping in dynamic relaxation", Int. J. Numer. Meth. Eng., 4, 301-304.

Cassell, A.C. and Hobbs, R.E. (1976), "Numerical stability of dynamic relaxation analysis of non-linear structures", Int. J. Numer. Meth. Eng., 10, 1407-1410.

Cassell, A.C., Kinsey, P.J. and Sefton, D.J. (1968), "Cylindrical shell analysis by dynamic relaxation", Proc. ICE, 39, 75-84.

Day, A.S. (1965), “An introduction to dynamic relaxation”, The Engineer, 219, 218-221.

Felippa, C.A. (1982), "Dynamic relaxation under general increment control”, Math. Prog., 24, 103-133.

Felippa, C.A. (1999), Nonlinear Finite Element Methods, ASEN 5017, Course Material, http://kaswww.colorado. edu/courses.d /NFEMD/; Spring.

Frankel, S.P. (1950), "Convergence rates of iterative treatments of partial differential equations", Math. Tables Other Aids Comput., 4(30), 65-75.

Frieze, P.A., Hobbs, R.E. and Dowling, P.J. (1978), "Application of dynamic relaxation to the large deflection elasto-plastic analysis of plates", Comput. Struct., 8, 301-310.

Han, S.E. and Lee, K.S. (2003), "A study on stabilizing process of unstable structures by dynamic relaxation method", Comput. Struct., 80, 1677-1688.

Kadkhodayan, M. and Zhang, L.C. (1995), "A consistent DXDR method for elastic-plastic problems", Int. J. Numer. Meth. Eng., 38, 2413-2431.

Kadkhodayan, M., Alamatian, J. and Turvey, G.J. (2008), "A new fictitious time for the dynamic relaxation (DXDR) method”, Int. J. Numer. Meth. Eng., 74, 996-1018.

Kadkhodayan, M., Zhang, L.C. and Swerby, R. (1997), "Analysis of wrinkling and buckling of elastic plates by DXDR method", Comput. Struct., 65, 561-574.

Murphy, J., Ridout, D. and McShane, B. (1988), Numerical Analysis Algorithms and Computation, Ellis 
Horwood Limited, New York.

Otter, J.R.H. (1966), "Dynamic relaxation", Proc. ICE, 35, 633-656.

Papadrakakis, M. (1981), "A method for automatic evaluation of the dynamic relaxation parameters", Comput. Meth. Appl. Mech. Eng., 25, 35-48.

Pasqualino, I.P. and Estefan, S.F. (2001), "A nonlinear analysis of the buckle propagation problem in deepwater piplines", Int. J. Solids Struct., 38, 8481-8502.

Qiang, S. (1988), "An adaptive dynamic relaxation method for non-linear problems", Comput. Struct., 30, 855859.

Ramesh, G and Krishnamoorthy, C.S. (1993), "Post-buckling analysis of structures by dynamic relaxation", Int. J. Numer. Meth. Eng., 36, 1339-1364.

Ramesh, G. and Krishnamoorthy, C.S. (1994), "Inelastic post-buckling analysis of truss structures by dynamic relaxation method", Int. J. Numer. Meth. Eng., 37, 3633-3657.

Rezaiee-Pajand, M. and Alamatian, J. (2008), "Nonlinear dynamic analysis by dynamic relaxation method", Struct. Eng. Mech., 28(5), 549-570.

Rushton, K.R. (1968), "Large deflection of variable-tickness plates", Int. J. Mech. Sci., 10, 723-735.

Saka, M.P. (1990), "Optimum design of pin-jointed steel structures with practical applications", J. Struct. Eng., ASCE, 116, 2599-2619.

Shawi, F.A.N. and Mardirosion, A.H. (1987), "An improved dynamic relaxation method for the analysis of plate bending problems", Comput. Struct., 27, 237-240.

Timoshenko, S. and Woinowsky-Krieger, S. (1959), Theory of Plates and Shells, Mc-Graw-Hill Book Company.

Turvey, GJ. and Salehi, R.E. (2005), "Annular sector plates : Comparison of full-section and layer yield prediction", Comput. Struct., 83, 2431-2441.

Turvey, G.J. and Salehi, R.E. (1990), "DR large deflection analysis of sector plates", Comput. Struct., 34, 101112.

Ugural, A.C. and Fenster, S.K. (1987), Advanced Strength and Applied Elasticity. Elsevier, New York.

Undewood, P. (1983), "Dynamic relaxation. computational method for transient analysis", Chapter 5, 245-256.

Welsh, A.K. (1967), "Discussion on dynamic relaxation", Proc. ICE, 37, 723-750.

Wood, R.D. (2002), "A simple technique for controlling element distortion in dynamic relaxation form-finding of tension membranes", Comput. Struct., 80, 2115-2120.

Wood, W.L. (1971), "Note on dynamic relaxation", Int. J. Numer. Meth. Eng., 3, 145-147.

Zhang, L.C. and Yu, T.X. (1989), "Modified adaptive dynamic relaxation method and its application to elasticplastic bending and wrinkling of circular plates", Comput. Struct., 34, 609-614.

Zhang, L.C., Kadkhodayan, M. and Mai, Y.W. (1994), "Development of the maDR method", Comput. Struct., 52, $1-8$.

Zienkiewicz, O.C. and Lohner R. (1985), "Accelerated relaxation or direct solution future prospects for FEM", Int. J. Numer. Meth. Eng., 21, 1-11. 


\section{Appendix 1}

The inverse prove is used for showing the validity of Eq. (40). Assume that

$$
2\left(1+a_{i i}\right)<\frac{2 \tau^{n+1} \tau^{n}}{2 m_{i i}+\tau^{n} c_{i i}} \sum_{j=1}^{q}\left|S_{i j}\right| \quad i=1,2, \ldots, q
$$

Substituting Eq. (39) into (A1-1) leads to

$$
\lambda_{i U}^{B S}+\lambda_{i L}^{B S}<\frac{2 \tau^{n+1} \tau^{n}}{2 m_{i i}+\tau^{n} c_{i i}} \sum_{j=1}^{q}\left|S_{i j}\right| \quad i=1,2, \ldots, q
$$

The Gerschgörin theory presents the lower bound of each eigenvalue as follows

$$
\lambda_{i L}^{B S} \geq \frac{2 \tau^{n+1} \tau^{n}}{2 m_{i i}+\tau^{n} c_{i i}} S_{i i} \geq 1 \quad i=1,2, \ldots, q
$$

If both sides of Eq. (A1-2) are multiplied by -1 and result is added to the Eq. (A1-3), the following result is achieved

$$
\lambda_{i U}^{B S}<\frac{2 \tau^{n+1} \tau^{n}}{2 m_{i i}+\tau^{n} c_{i i}} \sum_{\substack{j=1 \\ j \neq i}}^{q}\left|S_{i j}\right| \quad i=1,2, \ldots, q
$$

On the other hand, Eq. (18) presents the following relationship for $\lambda_{i U}^{B S}$

$$
\lambda_{i U}^{B S} \leq \frac{2 \tau^{n+1} \tau^{n}}{2 m_{i i}+\tau^{n} c_{i i}} \sum_{j=1}^{q}\left|S_{i j}\right| \quad i=1,2, \ldots, q
$$

Comparing Eq. (A1-4) with (A1-5) shows that Eq. (A1-4) limits the acceptable domain for $\lambda_{i U}^{B S}$. Therefore, assumption of Eq. (A1-1) is not suitable. This property can also be discussed from structural analysis point of view. In linear problem, the diagonal stiffness entry is greater than or equal to the absolute sum of the rest row (Eq. (29)). Combining Eq. (29) and (A1-4) leads to

$$
\lambda_{i U}^{B S}<\frac{2 \tau^{n+1} \tau^{n}}{2 m_{i i}+\tau^{n} c_{i i}} S_{i i} \quad i=1,2, \ldots, q
$$

Eq. (A1-6) is not valid because each eigenvalue of $[B]^{-1}[S]$ must be lied in domain I, distinguished by Eq. (18) (as proved in this paper, Eqs. (20)-(30)). Therefore, assumption of Eq. (A1-1) is not valid. As a result, Eq. (40) is correct.

\section{Appendix 2}

Mathematically, it is known that the capability of an iterative method to yield converged results depends on the magnitude of its convergence rank. One of the conventional methods for determining the convergence rank uses Taylor series. In this method, the degree of the first non-zero derivative in the iterative relationship is usually identified as the convergence rank (Murphy et al. 1988), as shown in the following relationship

$$
\varepsilon^{n+1}=\varepsilon^{n} g^{\prime}(\alpha)+\frac{\left(\varepsilon^{n}\right)^{2}}{2 !} g^{\prime \prime}(\alpha)+\ldots+\frac{\left(\varepsilon^{n}\right)^{m}}{m !} g^{(m)}(\alpha)+\ldots
$$

Where, the quantities $\alpha$ and $\varepsilon^{n}$ are the real solution and the error in the $\mathrm{n}^{\text {th }}$ iteration, respectively $D^{n}=\alpha+\varepsilon^{n}$. The function $g$ is determined from the characteristics of the solution process (Ugural and Fenster 1987). The following equation is held

$$
D^{n+1}=g\left(D^{n}\right)
$$

Now, the convergence ranks of the mdDR method are determined. First, the iterative function $(g)$ is 
formulated from Eqs. (3) and (4) in the following form

$$
g(D)=D+\tau^{n+1}\left[\frac{2-c^{n} \tau^{n}}{2+c^{n} \tau^{n}} \dot{D}^{n-1 / 2}+\frac{2 \tau^{n}}{\left(2+c^{n} \tau^{n}\right) m^{n}} r^{n}\right]
$$

where the fictitious mass and damping quantities may be substituted from Eqs. (44) and (50), respectively. For a one degree of freedom system, these quantities can be simplified as follows

$$
\left\{\begin{array}{l}
m^{n}=\frac{\left(\tau^{n}\right)^{2} S_{T}}{2} \\
c^{n} \tau^{n}=\frac{2}{S_{T}} \sqrt{S_{G}\left(2 S_{T}-S_{G}\right)}
\end{array}\right.
$$

where $S_{T}$ and $S_{G}$ are the tangent and secant stiffness, respectively. Substituting (A2-4) in to the (A2-3), the function $g$ becomes

$$
g_{N}(D)=D+\frac{S_{T}-\sqrt{S_{G}\left(2 S_{T}-S_{G}\right)}}{S_{T}+\sqrt{S_{G}\left(2 S_{T}-S_{G}\right)}} \tau^{n} D^{n-1 / 2}+\frac{2 r^{n}}{S_{T}+\sqrt{S_{G}\left(2 S_{T}-S_{G}\right)}}
$$

where $\dot{D}^{n-1 / 2}$ denotes the nodal velocity during the last iteration and has a constant value. In linear behavior $\left(S_{T}=S_{G}\right)$, the function $g$ can be simplified as follows

$$
g_{L}(D)=D+\frac{r^{n}}{S_{T}}
$$

On the other hand, the first derivatives of functions $g_{L}$ and $g_{N}$ with respect to displacement (D), can be found as follows

$$
\begin{gathered}
g_{N}^{\prime}(\alpha)=\frac{2\left(S_{G}-S_{T}\right)\left[S_{T} \frac{\partial S_{G}}{\partial D}-S_{G} \frac{\partial S_{T}}{\partial D}\right]}{\sqrt{S_{G}\left(2 S_{T}-S_{G}\right)}} \tau^{n} \dot{D}^{n-1 / 2}\left|-\frac{r^{n} \frac{\partial S_{T}}{\partial D}}{\left(S_{T}\right)^{2}}\right|_{\alpha} \\
=\left.\frac{2\left(S_{G}-S_{T}\right)\left[S_{T} \frac{\partial S_{G}}{\partial D}-S_{G} \frac{\partial S_{T}}{\partial D}\right]}{\sqrt{S_{G}\left(2 S_{T}-S_{G}\right)}} \tau^{n} \dot{D}^{n-1 / 2}\right|_{\alpha} \neq 0 \\
g_{L}^{\prime}(D)=-\left.\frac{r^{n} \frac{\partial S_{T}}{\partial D}}{S_{T}^{2}}\right|_{\alpha}=0
\end{gathered}
$$

Here, the primes denote derivatives with respect to the displacement. Because of the non-zero first derivative for the function $g_{N}$, the convergence rank of the mdDR method in nonlinear analysis will be equal to unity. Also, the second derivative of $g_{L}$ is

$$
g_{L}^{\prime \prime}(D)=-\left.\frac{r^{n}\left[\frac{\partial^{2} S_{T}}{\partial D^{2}} S_{T}-2\left(\frac{\partial S_{T}}{\partial D}\right)^{2}\right]-\left(S_{T}\right)^{2} \frac{\partial S_{T}}{\partial D}}{\left(S_{T}\right)^{3}}\right|_{\alpha}=\left.\frac{\frac{\partial S_{T}}{\partial D}}{S_{T}}\right|_{\alpha}
$$

The tangent stiffness remains constant during the analysis for linear problems. Hence

$$
\frac{\partial S_{T}}{\partial D}=\frac{\partial^{2} S_{T}}{\partial D^{2}}=\ldots=\frac{\partial^{m} S_{T}}{\partial D^{m}}=\ldots=0
$$


Therefore, from Eqs. (A2-9) and (A2-10), the second derivative of $g_{L}$ is zero. It is easy to show that all of the subsequent derivatives for $g_{L}$ will be equal to zero. Thus, it can be concluded that the convergence rank of the mdDR method is infinite for linear problems. It should be reminded that the convergence ranks of the DR method for both linear and nonlinear problems are equal to unity (Kadkhodayan et al. 2008). 